ARTICLE

\title{
Sugar phosphate activation of the stress sensor elF2B
}

Qi Hao ${ }^{1,6}$, Jin-Mi Heo, ${ }^{1,5,6}$, Boguslaw P. Nocek² ${ }^{2}$ Kevin G. Hicks (1) ${ }^{3}$, Vincent S. Stoll ${ }^{2}$, Clint Remarcik , Sean Hackett (10) 1, Lauren LeBon (10) 1, Rinku Jain ${ }^{2}$, Dan Eaton', Jared Rutter (10 ${ }^{3,4}$, Yao Liang Wong (1) ${ }^{1,7 凶} \&$ Carmela Sidrauski iD 1,7凶

The multi-subunit translation initiation factor elF2B is a control node for protein synthesis. elF2B activity is canonically modulated through stress-responsive phosphorylation of its substrate elF2. The elF2B regulatory subcomplex is evolutionarily related to sugarmetabolizing enzymes, but the biological relevance of this relationship was unknown. To identify natural ligands that might regulate eIF2B, we conduct unbiased binding- and activitybased screens followed by structural studies. We find that sugar phosphates occupy the ancestral catalytic site in the elF2B $\alpha$ subunit, promote elF2B holoenzyme formation and enhance enzymatic activity towards elF2. A mutant in the elF2B $\alpha$ ligand pocket that causes Vanishing White Matter disease fails to engage and is not stimulated by sugar phosphates. These data underscore the importance of allosteric metabolite modulation for proper elF2B function. We propose that elF2B evolved to couple nutrient status via sugar phosphate sensing with the rate of protein synthesis, one of the most energetically costly cellular processes.

\footnotetext{
${ }^{1}$ Calico Life Sciences LLC, South San Francisco, CA, USA. ${ }^{2}$ Research \& Development, AbbVie, North Chicago, IL, USA. ${ }^{3}$ Department of Biochemistry, University of Utah School of Medicine, Salt Lake City, UT, USA. ${ }^{4}$ Howard Hughes Medical Institute, University of Utah School of Medicine, Salt Lake City, UT, USA. ${ }^{5}$ Present address: Loxo Oncology at Lilly, South San Francisco, CA, USA. ${ }^{6}$ These authors contributed equally: Qi Hao, Jin-Mi Heo. ${ }^{7}$ These authors jointly

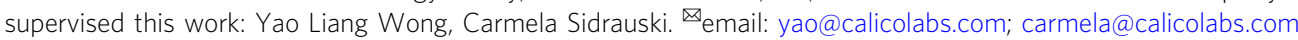


E ukaryotic translation initiation factor $2 \mathrm{~B}$ (eIF2B) is the decameric guanine nucleotide exchange factor (GEF) for the GTPase eukaryotic translation initiation factor 2 (eIF2). The essential function of these two complexes in cells is the delivery of the initiating methionine to the ribosome to allow protein synthesis. Upon delivery of the initiator Met-tRNA $A_{i}$ to the site of translation initiation, bound GTP is hydrolyzed and eIF2 is released from the scanning ribosome. eIF2B catalyzes GDP-GTP exchange on eIF2, enabling rebinding of Met-tRNA $A_{i}$ to allow for a new cycle of mRNA translation initiation ${ }^{1}$.

Because of the energetic and biosynthetic costs of protein synthesis, translation initiation is a highly regulated process. Four kinases respond to diverse stresses by phosphorylating the $a$ subunit of eIF2, converting it from a substrate into a tightbinding competitive inhibitor of eIF2 $\mathrm{B}^{2,3}$. The resulting decrease in formation of the active eIF2-GTP-Met- $R_{\text {RNA }}$ ternary complex reduces translation initiation and triggers the Integrated Stress Response (ISR). Its activation attenuates bulk protein synthesis while stimulating translation of a subset of transcripts, such as the transcription factor ATF4, that promote adaptation under stress conditions $^{4-6}$. Both of these ISR effects are important for cells to adapt to acute stress conditions but may lead to dysfunction when chronically engaged.

eIF2B is an enzyme of considerable complexity, comprising five different subunits arranged in a two-fold symmetric structure to form a decamer ${ }^{7-10}$. Two catalytic subcomplexes, each comprised of a $\gamma$ and $\varepsilon$ subunit, decorate the central regulatory core, which is a heterohexamer composed of two $\alpha, \beta$ and $\delta$ subunits. We and others demonstrated that small molecule eIF2B activators bind at the axis of symmetry, acting as molecular staplers that stabilize and activate the decameric holoenzyme to attenuate the $\mathrm{ISR}^{9-13}$. Furthermore, we showed that partial loss-of-function mutations in eIF2B that cause Vanishing White Matter (VWM), a devastating neurodegenerative disease, destabilize the eIF2B decamer and reduce its GEF activity. Stabilization of the decamer with eIF2B activators rescued the GEF activity of these mutations both in vitro and in vivo, revealing the importance of the decameric complex for normal eIF2B function ${ }^{13}$.

Curiously, the eIF2B regulatory core is related to an ancient family of homo-hexameric sugar phosphate-metabolizing enzymes, with conservation observed between $\operatorname{eIF} 2 \mathrm{~B} \alpha / \beta / \delta$ and the archaeal ribose-1,5-bisphosphate isomerase (RBPI) ${ }^{14-16}$. Why was a metabolic enzyme co-opted to serve as the core for a multisubunit GEF? One enticing hypothesis is that this might enable direct coupling of nutrient availability to protein synthesis, the most energy-intensive process in the cell. Sugar phosphorylation is the first step in the catabolism of carbohydrates to drive ATP generation and biomass accumulation. The eIF2a kinase GCN2 senses amino acid availability, whereas the other kinases evolved to detect non-nutrient stressors ${ }^{17,18}$. None of these kinases sense carbon or ATP abundance, and phosphorylation itself is an energy-consuming act, thus an ancient and direct role for eIF2B in energy-sensing is compelling.

We note that the origins of our hypothesis are over 30 years old. Gross et al. proposed that eIF2B activity could be matched to energy availability, based on observations that glucose-6phosphate was required to maintain mRNA translation rates in cell lysates ${ }^{19}$. Contemporaneously, Wahba et al. suggested that pyridine dinucleotides $\left(\mathrm{NAD}^{+} / \mathrm{NADP}^{+} / \mathrm{NADPH}\right)$ served a role in controlling eIF2 ternary complex turnover ${ }^{20}$. These early works were hampered by a lack of readily available metabolites to test, and an inability to use fully reconstituted in vitro systems. Eventually, the community directed its attention to studying the regulation of eIF2B by phosphorylated eIF2 $\alpha$. More recently, Kuhle et al. used isothermal titration calorimetry (ITC) to demonstrate binding of the monophosphate ribonucleotides
AMP and GMP to recombinant $C$. thermophilum eIF2Ba ${ }^{16}$. This prompted us to revisit the concept of nutrient status as a regulator of the eIF2B-eIF2 axis, with a focus on direct sensing by eIF2B.

In this work, we conduct two orthogonal unbiased screens for potential metabolite ligands of eIF2B, identifying sugar phosphates as eIF2B $\alpha$ binders and enzymatic activators. Using cryoEM and X-ray crystallography, we elucidate structures of sugar phosphates bound to eIF2B and demonstrate that they occupy the ancestral catalytic pocket within the eIF2Ba subunit. We further show that sugar phosphates stabilize the decameric holoenzyme and enhance its GEF activity similar to synthetic eIF2B activators. However, these natural metabolites exert their effect by engaging the eIF2 $\mathrm{Ba}_{2}$ dimer, rather than by bridging the $\operatorname{eIF} 2 \mathrm{~B}(\beta / \delta)_{2}$ tetramer interface like ISRIB. Unlike the recently demonstrated activity of ISRIB as an antagonist of phosphorylated eIF2 binding ${ }^{21,22}$, the sugar phosphate mechanism is primarily driven by its ability to decamerize eIF2B. We generate point mutations in the eIF2Ba sugar-binding pocket, one of which is known to cause VWM disease. These mutations abolish metabolite binding and the concomitant increases in decamer stabilization and activity, raising the possibility that impaired sugar phosphate sensing by eIF2B may contribute to disease etiology. In sum, our results underscore the importance of sugar phosphate metabolites in modulation of eIF2B activity.

\section{Results}

An unbiased screening approach identifies sugar phosphates as ligands of eIF2Ba. Beginning with our hypothesis that eIF2B may be a direct nutrient sensor, we reasoned that the eIF $2 \mathrm{~B} \alpha / \beta / \delta$ regulatory subunits could be ideal candidates for an unbiased metabolite screen by virtue of their homology to aforementioned sugar-metabolizing enzymes. To this end, we employed mass spectrometry integrated with equilibrium dialysis for the discovery of allostery systematically (MIDAS) ${ }^{23}$. Due to the high protein concentrations required for the MIDAS approach, we focused our efforts on the small $34 \mathrm{kDa}$ eIF2Ba subunit, which can be purified in large quantities and forms a stable homodimer $\left(\mathrm{eIF} 2 \mathrm{Ba}_{2}\right)$ in solution. By contrast, eIF2B $\beta / \delta$ form a $227 \mathrm{kDa}$ heterotetrameric subcomplex with the $\gamma$ and $\varepsilon$ subunits ${ }^{8}$.

MIDAS screening of 412 naturally occurring human metabolites against eIF2Ba $a_{2}$ yielded 16 that were enriched or depleted using cutoffs of $\log _{2}$ (fold-change) $<-0.25$ or $>0.25, \mathrm{q}<0.1$ (Fig. 1a, b, Supplementary Data 1). Enriched hits are metabolites that are more concentrated in the eIF2Ba-containing chamber, consistent with direct physical interaction. Depleted hits in MIDAS are more complicated to interpret and can indicate tight physical interactors, covalent modifications of metabolites to the target protein, or enzymatic substrates that are consumed in the protein chamber at a rate faster than the metabolite diffusion rate across the chambers, leading to an apparent loss of the metabolite. The amino acid tyrosine was the most enriched metabolite identified, and its derivative iodotyrosine was also significantly enriched. Interestingly, 11/16 of the hits were sugars, of which 8 were sugar phosphates (including ribonucleotides) similar to the substrate of RBPI, ribose-1,5-bisphosphate.

To orthogonally validate the MIDAS hits, we performed differential scanning fluorimetry on a subset of metabolites to assess their effect on the thermal stability of eIF2Ba $a_{2}$ (Fig. 1c, Supplementary Fig. 1a). We also assayed a number of sugar phosphates that did not reach statistical significance in MIDAS, recognizing that they may not have met the significance threshold due to the inability of our mass spectrometry method to distinguish between identical sugar masses (glucose, mannose, galactose, and fructose), resulting in signal splitting across 7 different sugar phosphate isomers. The MIDAS hits increased the 
a

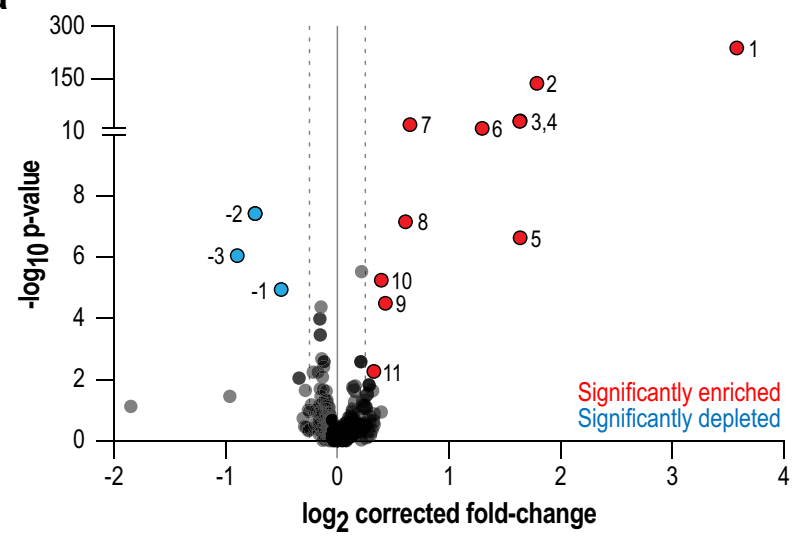

b

\begin{tabular}{|c|l|}
\hline$\#$ & Metabolite \\
\hline 1 & L-tyrosine \\
\hline 2 & Uridine monophosphate \\
\hline 3 & 2-deoxyribose 5-phosphate \\
\hline 4 & Nicotinamide riboside \\
\hline 5 & Guanosine monophosphate \\
\hline 6 & Cytidine monophosphate \\
\hline 7 & lodotyrosine \\
\hline 8 & Inosine monophosphate \\
\hline 9 & Benzoic acid \\
\hline 10 & N-acetyl-D-glucosamine/galactosamine \\
\hline 11 & Tyramine \\
\hline-3 & Galactitol \\
\hline-2 & D-fructose/glucose-1,6-bisphosphate \\
\hline-1 & 5-hydroxyl-L-tryptophan \\
\hline
\end{tabular}

C
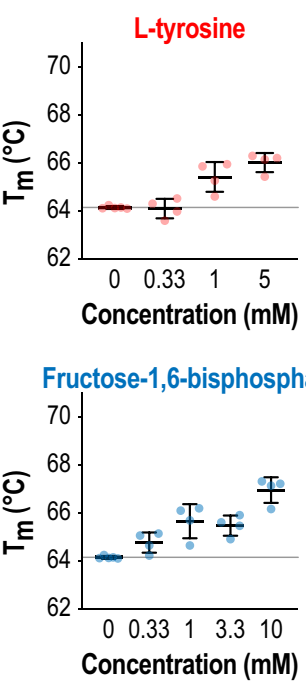
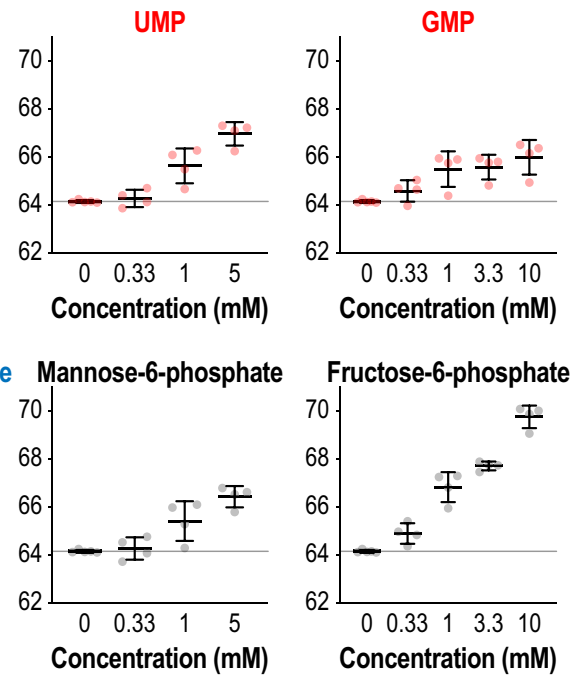

d

d elF2B $\alpha+$ fructose-6-phosphate

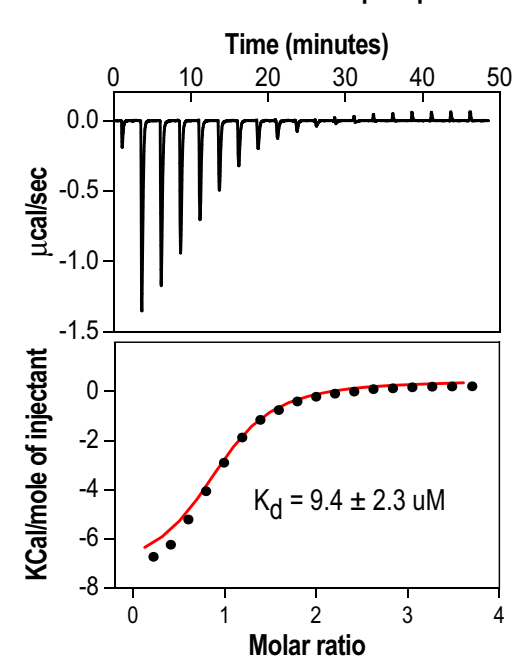

Fig. 1 Unbiased screening by MIDAS identifies putative ligands that interact with elF2B $\boldsymbol{\alpha}$. a Volcano plot of metabolites analyzed in MIDAS, comparing the fold-change between the protein chamber and metabolite chamber. Red indicates metabolites that were significantly enriched in the protein-containing chamber, whereas blue indicates metabolites that were significantly depleted $(q<0.1)$ based on a two-tailed Wald test. The full data are available as Supplementary Data 1. b All 16 significant hits from the MIDAS binding screen numbered in a are tabulated. c Differential scanning fluorimetry of elF2B $\alpha$ in combination with selected metabolites in dose-response. Metabolite binding increased the $T_{m}$ of elF2B $\alpha$. Bars are mean \pm standard deviation of $n=4$ independent experiments. Color coding in $\mathbf{b}, \mathbf{c}$ are as in $\mathbf{a} . \mathbf{d} \mathrm{K}_{\mathrm{d}}$ of the elF2B $\alpha-F 6 \mathrm{P}$ interaction measured by ITC. The upper subpanel shows the baselinesubtracted thermogram. The bottom subpanel represents the binding isotherm, with the red line indicating the fit curve.

melting temperature $\left(\mathrm{T}_{\mathrm{m}}\right)$ of $\mathrm{eIF} 2 \mathrm{Ba}_{2}$ in a concentrationdependent manner. Remarkably, fructose-6-phosphate (F6P), which was not a MIDAS hit, induced the largest $\mathrm{T}_{\mathrm{m}}$ shift of $6{ }^{\circ} \mathrm{C}$ (Fig. 1c).

Given the marked increase in thermal stability induced by F6P and its key position in central carbon metabolism, we examined its interaction with eIF2Ba in more detail. ITC measurements showed that it binds to eIF2Ba with $\mathrm{K}_{\mathrm{d}}=9.4 \pm 2.3 \mu \mathrm{M}$ (Fig. 1d). By contrast, the affinity of eIF2Ba for the F6P isomer mannose-6phosphate (M6P) was considerably weaker, with $\mathrm{K}_{\mathrm{d}}=282 \pm 35 \mu \mathrm{M}$ (Supplementary Fig. 1b). Although there is considerable uncertainty about free intracellular metabolite concentrations (and in vivo values are likely to be highly dependent on cell type, nutrient status, and other factors), these values are consistent with reported values of many sugar phosphates in the $1-100 \mu \mathrm{M}$ range $^{24}$. In summary, the unbiased screen and ensuing characterization indicated that sugar phosphates are ligands for eIF2B $\alpha$ at physiologically relevant concentrations.
Unbiased activity-based screening identifies $5^{\prime} / 6^{\prime}$ sugar phosphates as eIF2B activators. The primary function of eIF2B is to catalyze the exchange of GDP for GTP on its cognate substrate eIF2. Our results thus far were highly suggestive of a bona fide interaction between sugar phosphates and eIF2Ba, but MIDAS does not report on the effect of binding on eIF2B function. We and others previously described fluorescence-based assays to monitor eIF2B GEF activity on eIF2 ${ }^{10,12,25}$. Hence, we used an arrayed version of the MIDAS metabolite library to conduct an orthogonal unbiased screen based on modulation of eIF2B activity (Fig. 2a, Supplementary Data 2).

At a screening concentration of $10 \mu \mathrm{M}$, the top 10 activators of eIF2B GEF activity were all sugars with a $5^{\prime}$ or $6^{\prime}$ phosphate moiety, consistent with the structure of the ancestral substrate of this complex (Fig. 2b, c). Some metabolites, such as F6P and M6P, were represented twice in the physical screening library, and the activity screen positioned both replicates of each metabolite within the most activating hits, increasing our confidence of their 
a



b

Top 10 activators

\begin{tabular}{|l|c|}
\hline \multicolumn{1}{|c|}{ Metabolite } & GDP release $\mathbf{t}_{\mathbf{1 / 2}}$ (mins) \\
\hline Fructose-6-phosphate & 5.8 \\
\hline Uridine monophosphate & 5.8 \\
\hline Fructose-6-phosphate & 6.2 \\
\hline Adenosine diphosphate & 6.4 \\
\hline Coenzyme A & 6.5 \\
\hline Mannose-6-phosphate & 6.6 \\
\hline 2-deoxyribose 5-phosphate & 6.8 \\
\hline Adenosine monophosphate & 7 \\
\hline Glucose-6-phosphate & 7.2 \\
\hline Mannose-6-phosphate & 7.4 \\
\hline
\end{tabular}

C
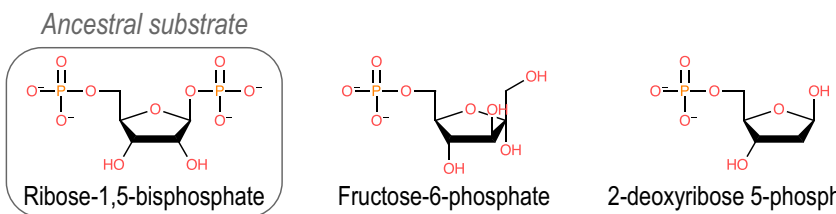

2-deoxyribose 5-phosphate

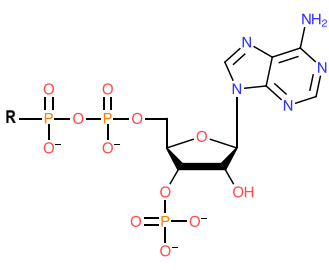

Coenzyme A
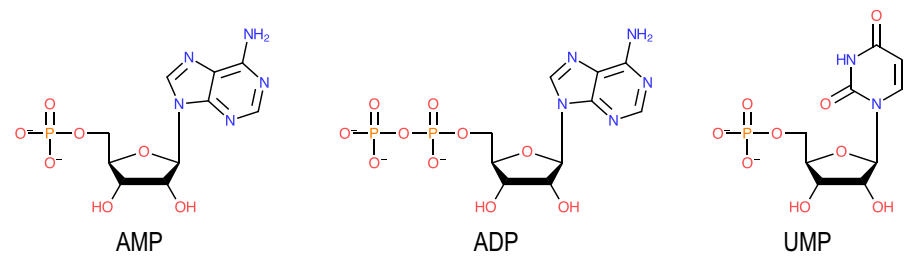

d
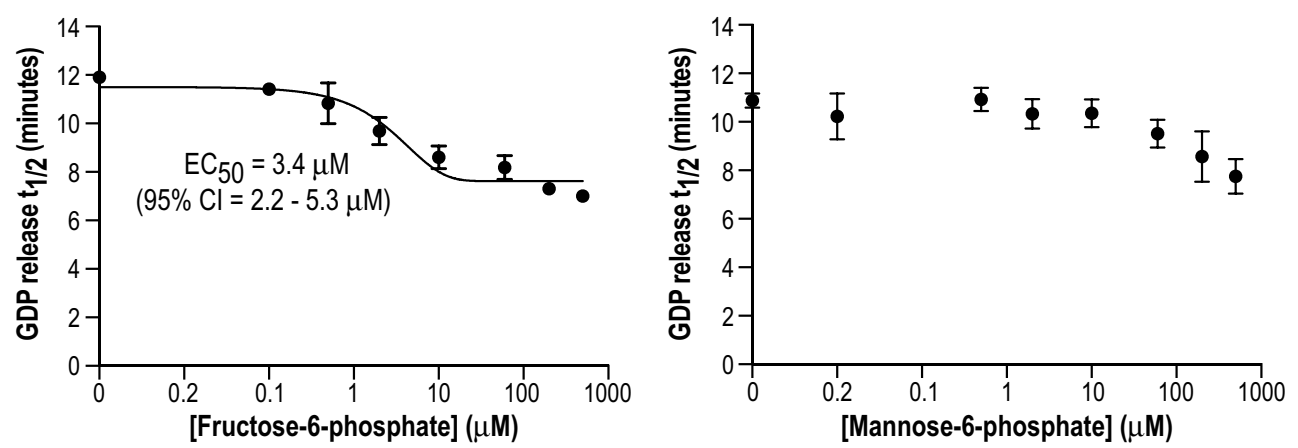

Fig. 2 Unbiased activity-based screening identifies $5^{\prime} / \mathbf{6}^{\prime}$ sugar phosphates as elF2B activators. a Plot of individual metabolites arrayed against GDP release $t_{1 / 2}$ derived from the GEF activity assay. Based on the basal activity of elF2B under these assay conditions, a $t_{1 / 2}$ cutoff of $<10$ min was used to define activators (green) and a cutoff of $>20$ min was used to define inhibitors (orange). $\mathbf{b}$ List of the top 10 activators from the screen, ordered by GDP release $t_{1 / 2}$. The full data are available as Supplementary Data 2. c Structures of the top 10 activators from the screen, compared to the substrate of archaeal RBPI. The sugar ring and $5^{\prime} / 6^{\prime}$ phosphate moiety are common structural motifs. d GDP release $t_{1 / 2}$ of elF2B using increasing concentration of F6P or M6P. Only the elF2B + F6P data could be fit to a dose-response curve with $R_{2}>0.9$. Each point represents mean \pm standard deviation of 3 technical replicates.

significance. UMP, a strong hit in MIDAS, was also a hit in this functional assay screen, together with other ribonucleotides such as AMP and ADP. We attribute their effect to the ribophosphate portion of the molecule, rather than the base group (see structure data below). Tyrosine, which was a prominent ligand identified by MIDAS, did not show an appreciable effect on eIF2B GEF activity in vitro, thus we did not pursue it further. Whether it has a regulatory role in complex assembly in cells remains to be determined.

We further investigated the stimulatory effects of F6P and M6P on GEF activity by performing dose-response experiments with eIF2B (Fig. 2d). As suggested by the thermal shift and ITC results
(Fig. 1c, d, Supplementary Fig. 1b), F6P is a more potent activator $\left(\mathrm{EC}_{50}=3.4 \mu \mathrm{M}\right)$ of eIF2B activity than M6P. Our activity screen also identified a set of inhibitors, including bile salts and a variety of acidic compounds (Fig. 2a, Supplementary Data 2). None of the identified inhibitors appeared to interact with eIF2Ba based on MIDAS, but we cannot rule out potential interactions with the eIF2B holoenzyme. Nevertheless, there was no obvious structural commonality between inhibitors, likely because there are many possible ways to non-specifically interfere with an enzymatic assay, but far fewer ways to enhance activity. Thus, we constrained our further investigation to understanding the mechanism of the sugar phosphates as activators. 


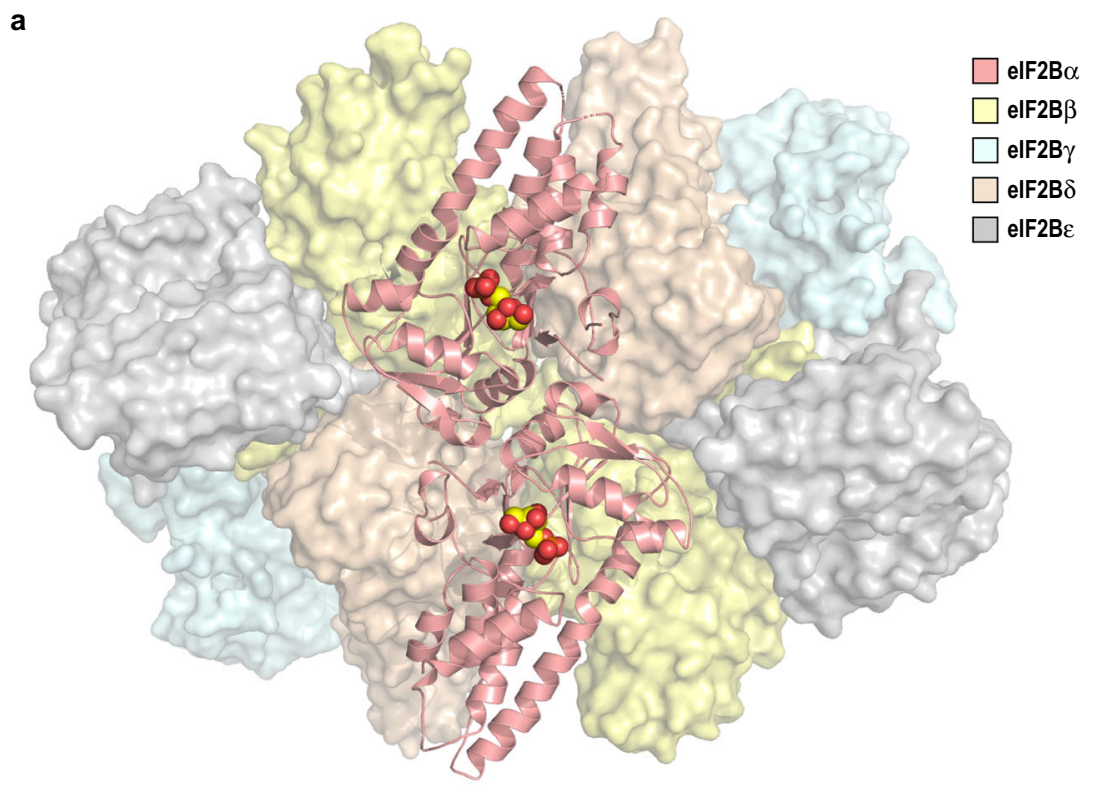

b

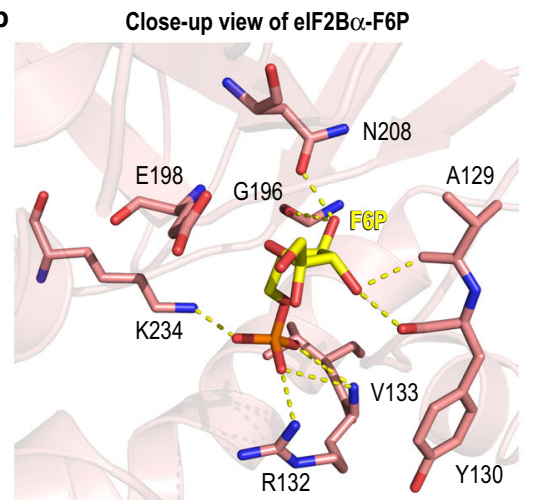

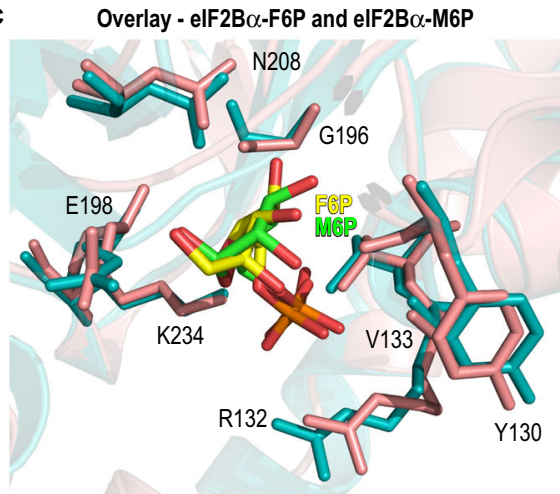

Fig. $32.9 \AA$ cryo-EM structure of elF2B in complex with F6P. a Overall structure of the elF2B-F6P complex with elF2B $\alpha$ in cartoon representation and elF2B $\beta / \delta / \gamma / \varepsilon$ represented as surfaces (PDB 7KMF). The single F6P molecule bound within each elF2B $\alpha$ monomer is shown as space-filling spheres. b Close-up view of the elF2B $\alpha$ metabolite binding pocket, with residues contributing to the $\mathrm{F} 6 \mathrm{P}$ interaction shown in stick representation. $\mathrm{H}$-bonds are represented by dashed yellow lines. c Overlay of the sugar phosphate binding pockets in the elF2B-F6P cryo-EM structure (pink) and the elF2B $\alpha-M 6 P$ crystal structure (PDB 7KMA; teal). F6P and M6P are shown as yellow and green sticks, respectively.

Sugar phosphates bind to the evolutionarily conserved substrate binding pocket in eIF2Ba. Motivated by our biochemical evidence that sugar phosphates are activators of eIF2B, we determined a $2.9 \AA$ cryo-EM structure of eIF2B bound to F6P, the highest affinity identified ligand (Supplementary Figs. 2 and 3). The eIF2B-F6P complex shares the same overall architecture as previously reported eIF2B structures (Fig. 3a) $)^{9,10}$. F6P occupies an inter-domain cavity within each eIF2Ba monomer (chains $\mathrm{G}$ and $\mathrm{H}$ ), which is the conserved metabolite binding pocket in the ancestral $\operatorname{eIF} 2 \mathrm{~B} \alpha / \beta / \delta$ homologs ${ }^{16,26}$. This pocket is on the opposite face of the eIF2B holoenzyme from the previously described ISRIB binding pocket that sits at the symmetric eIF2B $\beta$ / $\delta$ interface. Protein-ligand interactions in the $\mathrm{F} 6 \mathrm{P}$ pocket are mediated by both the sugar and phosphate moieties. G196, A129, Y130, E198, and N208 form H-bonds with the F6P pyranose ring, while the positively charged residues R132 and K234 coordinate the phosphate group (Fig. $3 \mathrm{~b}$ ). The $1^{\prime}$ position of F6P is exposed to solvent, suggesting a way by which bulkier ribonucleotides (e.g. UMP and AMP) could be accommodated in this site.

Notably, we did not observe electron density for any ligands within the eIF2B $\beta$ or eIF2B $\delta$ metabolite pockets, despite their homology to eIF2Ba. An examination of their amino acid sequences revealed that key residues involved in sugar ring coordination (E198 and N208) are conserved between eIF2Ba and two homologs from the same enzyme family with known metabolite binding activity, but not conserved in eIF2B $\beta / \delta$ (Supplementary Fig. 4a). This pattern is maintained in yeast eIF2B subunit sequences, suggesting that the functional divergence of the eIF $2 \mathrm{~B} \alpha / \beta / \delta$ paralogs is ancient and may have coevolved with the introduction of eIF2 phosphorylation as a mode of regulation. Consistent with our observation, ITC measurements showed that F6P did not interact appreciably with an $\operatorname{eIF} 2 \mathrm{~B}(\beta \delta \gamma \varepsilon)$ subcomplex (Supplementary Fig. $4 \mathrm{~b})$. An eIF2Ba homolog, human methylthioribose-1-phosphate isomerase (MTNA), binds to a sugar with a $1^{\prime}$ phosphate group. While residues in MTNA participating in sugar coordination are conserved with eIF2Ba, residues involved in phosphate coordination have diverged, likely due to the difference in phosphate group position (Supplementary Fig. 4a). We confirmed the importance of the phosphate position by demonstrating that eIF2Ba did not interact with the F6P isomer glucose-1-phosphate (G1P; Supplementary Fig. 4c).

To confirm that the binding mode for F6P is shared with other sugar phosphates, we determined the $2.7 \AA$ crystal structure of eIF2Ba bound to M6P (Supplementary Figs. 5 and 6). Comparison of the F6P and M6P structures revealed a metabolite 
recognition mechanism employing the same residues in the eIF2Ba pocket (Fig. 3c). Slight differences in bonding due to the larger size of the M6P sugar could account for the difference in affinity between F6P and M6P.

To identify potential changes in protein conformation upon sugar phosphate binding, we performed pairwise comparisons of eIF2B-F6P and eIF2Ba-M6P with the reported structures of apo eIF2B (PDB 7D46 ${ }^{22}$ ) and eIF2Ba (PDB 3ECS ${ }^{27}$ ). eIF2Ba-M6P aligned to apo eIF2Ba with r.m.s.d. $=0.44 \AA$, indicating high overall similarity (Supplementary Fig. 7a). Curiously, the metabolite binding site in the apo structure is occupied by $\mathrm{SO}_{4}$ molecules from the crystallization buffer. This could suggest that the site must be filled for optimal protein stability. The free eIF2B $\alpha$ structures also align with eIF2Ba in the context of the holoenzyme (with or without F6P) with r.m.s.d. $<0.9 \AA$, and interdomain orientations are not significantly changed (Supplementary Fig. 7b).

The holoenzyme structures in the absence and presence of F6P are also strikingly similar, with global r.m.s.d. $=1.6 \AA$. The most prominent difference is a uniform shift in the position of both eIF2B $\varepsilon$ subunits, wherein the $\beta$-sheet rich domain moves inwards towards the cognate eIF2B $\alpha$ subunit by $\sim 1.5-2 \AA$ (Supplementary Fig. 7c). This results in a slightly compacted conformation of the holoenzyme in the presence of F6P, although all major structural features are otherwise preserved. Together, our data indicate that sugar phosphates interact with eIF2B exclusively through the a subunit, and that conserved residues in eIF2Ba confer selective binding via both the sugar and phosphate moieties.

Sugar phosphate binding by the a subunit enhances eIF2B decamer formation. We previously showed that eIF2B GEF activity is correlated with the stability of the decameric holoenzyme ${ }^{12}$. Indeed, the small molecule eIF2B activator ISRIB functions by serving as a molecular stapler between two eIF2B $(\beta \delta \gamma \varepsilon)$ subcomplexes ${ }^{9,10}$. In nature, the eIF2Ba $\alpha_{2}$ dimer has an analogous function as it promotes dimerization of these subcomplexes by bridging the two-fold symmetric interface of the holoenzyme. We hypothesized that sugar phosphate binding might enhance the ability of eIF2 $\mathrm{Ba}_{2}$ to promote this dimerization event and thus activation of the complex.

To examine this possibility, we chose to directly assess the effect of F6P binding on eIF2B decamer formation. We generated lysate from wild-type (WT) HEK293T cells and subjected them to sucrose gradient centrifugation in the presence of different ligands to separate the various assembly states of eIF2B $\left(\alpha_{2}, \beta \delta \gamma \varepsilon\right.$ and $[\alpha \beta \delta \gamma \varepsilon]_{2}$ ). In this assay, we used a supraphysiological salt concentration $(400 \mathrm{mM} \mathrm{KCl})$ to increase our sensitivity to detect transitions between assembly states. Subcomplexes were tracked and quantified using antibodies directed against eIF2B $\alpha$ and eIF2B $\delta$. As previously reported, ISRIB caused eIF2B $\alpha$ and eIF2B $\delta$ to co-migrate into a denser portion of the gradient, indicating formation of the decameric holoenzyme (Fig. 4a, b, fractions 9-12, demarked by the red dashed lines) $)^{11}$. Remarkably, F6P induced stabilization of the eIF2B decamer comparable to ISRIB. We confirmed that the identity of the sugar phosphate is important by showing that G1P, an inactive metabolite in the enzymatic assay, did not affect the migration of eIF2B subunits (Supplementary Fig. 8a-c). Migration of eIF3a, which is a constituent of another high molecular weight translation initiation complex, was not affected by any of the treatments, confirming the specificity of ISRIB and F6P.

As an orthogonal approach to assess complex formation, we performed size-exclusion chromatography on recombinant eIF2B subunits in the presence and absence of ISRIB or F6P (Supplementary Fig. 9a, b). As expected, ISRIB enhanced formation of an $(\beta \delta \gamma \varepsilon)_{2}$ octamer in the absence of eIF2B $\alpha$, and the $(\alpha \beta \delta \gamma \varepsilon)_{2}$ decamer in the presence of eIF2Ba. By contrast, and consistent with its exclusive binding to eIF2Ba, F6P was ineffective at promoting complex formation in the absence of eIF2Ba.

Based on structural analysis of the binding pocket, we generated a HEK293T cell line bearing a homozygous knock-in mutation of eIF2BaE198K, which we predicted would disrupt coordination of a ligand's sugar moiety. We validated our prediction by demonstrating that purified, recombinant eIF2$\mathrm{Ba}^{\mathrm{E} 198 \mathrm{~K}}$ exhibited 11-fold reduced affinity for F6P in an ITC assay (Supplementary Fig. 8d; compare with Fig. 1d). We attempted to generate a second knock-in cell line with another binding-disrupting mutant, eIF $2 \mathrm{Ba}^{\mathrm{N} 208 \mathrm{Y}}$, but were unsuccessful (see next section and Discussion). We subjected eIF2Ba ${ }^{\mathrm{E} 198 \mathrm{~K}}$ cell lysate to the same sucrose gradient analysis as above. As expected, ISRIB produced a shift to the decameric form in the mutant lysate, as it binds to the eIF2B $(\beta \delta \gamma \varepsilon)$ subcomplex (Fig. $4 c, d$ ). However, eIF2Ba $\alpha^{\mathrm{E} 198 \mathrm{~K}}$ complexes were unresponsive to F6P, confirming our hypothesis that sugar phosphate binding results in eIF2Ba-mediated holoenzyme formation.

Finally, we strengthened the mechanistic link between decamer formation and enzymatic activity by showing that the GEF activity of eIF2 $\mathrm{Ba}^{\mathrm{E} 198 \mathrm{~K}}$ lysate was boosted in the presence of ISRIB but not F6P (Fig. 4e). In these assays, we used a greater amount of mutant lysate than WT to achieve comparable enzymatic activity, as the mutation reduced the levels of eIF2B subunits, a phenomenon we have previously reported (Supplementary Fig. 8e, see Discussion) ${ }^{12}$. Interestingly, in WT eIF2B, we observed a similar boost in GEF activity using either ISRIB or F6P, but no additive effects when the two ligands were combined (Supplementary Fig. 9c). This is consistent with our hypothesis that these ligands converge upon a common pathway, i.e., eIF2B decamerization, to exert their activating effects.

Recent work showed that in addition to promoting eIF2B decamer formation, ISRIB can also enhance the enzymatic activity of this complex by allosterically antagonizing the interaction of eIF2B with its substrate-turned-inhibitor phospho-eIF2 21,22. Motivated by this exciting finding, we investigated whether F6P elicits a similar effect. To this end, we used HEK293T cells harboring a knock-in C-terminal FLAG tag at the endogenous eIF2B $\beta$ locus. We treated these cells with thapsigargin, an inhibitor of the endoplasmic reticulum $\mathrm{Ca}^{2+}$ ATPase, to generate an intracellular pool of phospho-eIF2a. Consistent with published work, we observed coimmunoprecipitation of phospho-eIF2 $\alpha$ with FLAG-tagged eIF2B that was significantly disrupted by ISRIB (Supplementary Fig. 10a, b) ${ }^{21,22}$. By contrast, F6P did not decrease the interaction between phospho-eIF2 $\alpha$ and eIF2B under these conditions, revealing a mechanistic difference between these two eIF2B activators.

Contrasting effects of sugar phosphate binding in two eIF2Ba VWM disease mutants. Among the conserved residues in the eIF2B $\alpha$ metabolite pocket, the sugar-coordinating N208 was of particular interest because an eIF2Ba ${ }^{\mathrm{N} 208 \mathrm{Y}}$ mutation has been reported in a patient with VWM disease ${ }^{28}$. To study the effect of this mutation, we chose to contrast it against another VWM mutation in eIF2Ba that we previously characterized, V183F, which does not reside in the binding pocket (Fig. 5a). Instead, eIF2 $\mathrm{Ba}^{\mathrm{V} 183 \mathrm{~F}}$ is a severe VWM mutation that localizes to the interface of the $\alpha_{2}$ homodimer and disrupts dimer formation as well as decamerization of the holoenzyme $8,12,29$.

We purified recombinant versions of both eIF2Ba VWM mutants and combined them with WT eIF2B $(\beta \delta \gamma \varepsilon)$ to reconstitute the mutant holoenzymes. As predicted, we observed that 
a
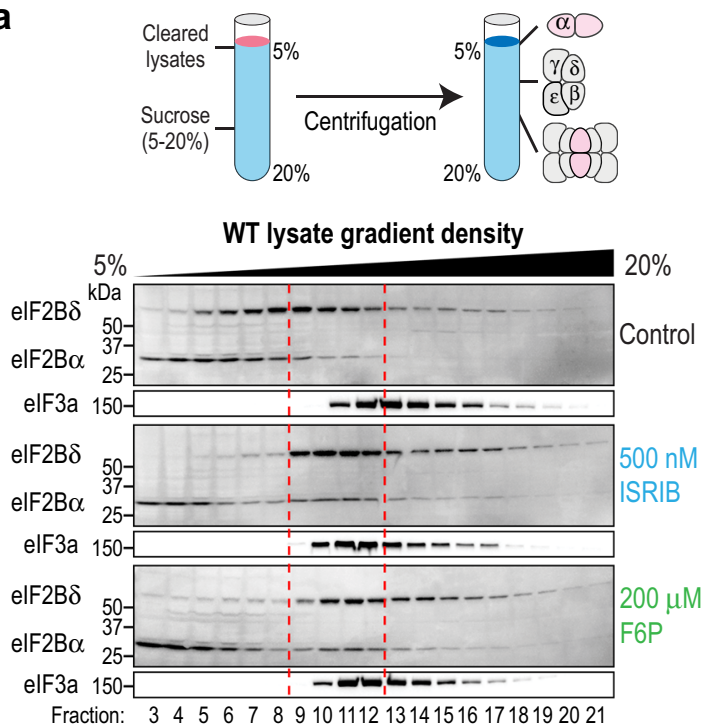

C

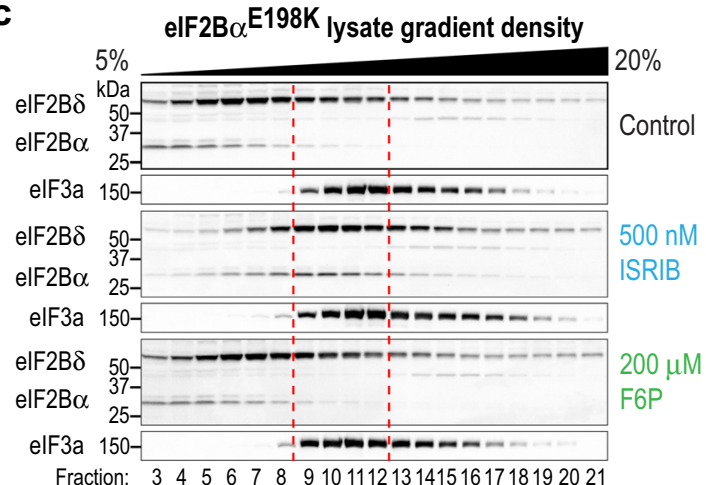

e

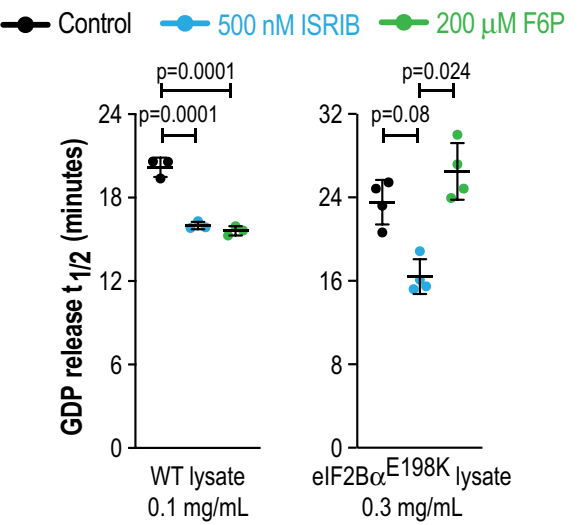

b
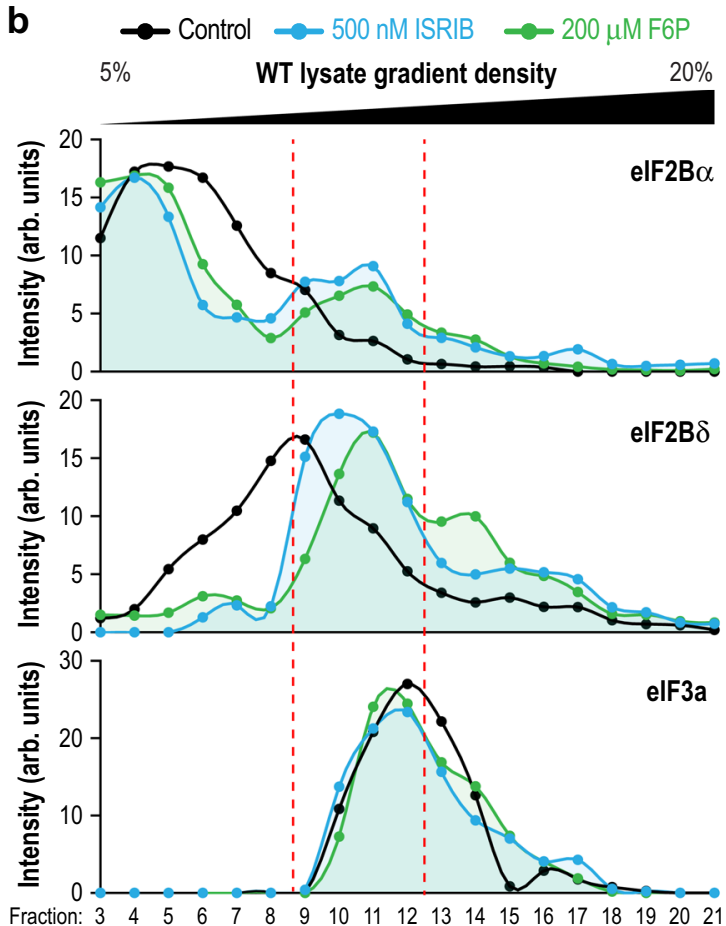

d


氷
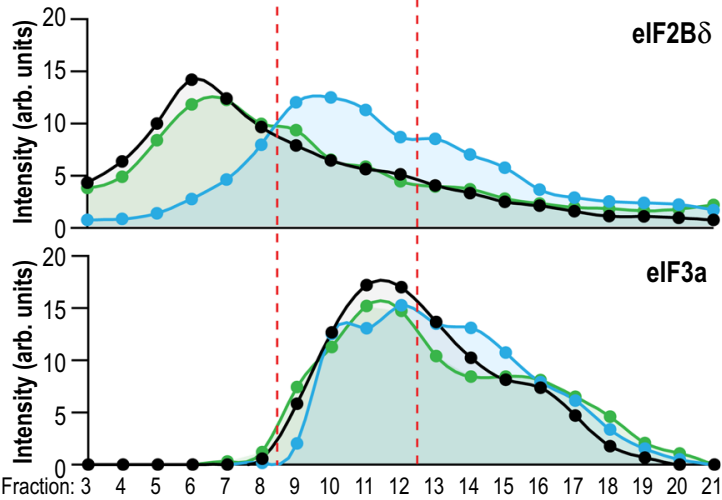

Fig. 4 Sugar phosphate binding by the $\alpha$ subunit enhances elF2B decamer formation. a-d elF2B complex assembly from WT and elF2B $\alpha$ E198K $H E K 293 T$ lysates treated with ISRIB (blue) or F6P (green) was monitored by sucrose gradient centrifugation. Fractions from the sucrose gradient were subjected to SDS-PAGE followed by immunoblotting with the indicated antibodies. elF3a was used as an internal control. Western blot data in $\mathbf{a}, \mathbf{c}$ are quantified in $\mathbf{b}, \mathbf{d}$, respectively. Data shown are representative of 2-3 replicates of each experiment. Bands were normalized by the total intensity of each subunit in its respective gradient. Dashed red lines demark the boundary of the decameric eIF2B peak. WT elF2B forms a decamer in the presence of both ISRIB and F6P. By contrast, elF2B $\alpha^{\text {E198K }}$ complexes respond to ISRIB but not F6P. e GDP release $t_{1 / 2}$ in a GEF assay using lysates from WT or elF2B $\alpha^{E 198 K}$ cells. WT lysate activity is stimulated by both ISRIB and F6P, whereas elF2B $\alpha$ E198K lysate does not respond to F6P. Bars are mean \pm standard deviation of $n=3$ independent experiments of 3 technical replicates each. Statistical significance was tested by one-way ANOVA with Tukey's multiple testing correction.

eIF2Ba ${ }^{\mathrm{N} 208 \mathrm{Y}}$ abrogates binding to F6P (Supplementary Fig. 11a), whereas eIF2BaV183F retains WT affinity for this ligand $\left(\mathrm{K}_{\mathrm{d}}=6.3 \pm 0.7 \mu \mathrm{M}\right.$; Supplementary Fig. 11b). The basal GEF activity of eIF2B reconstituted with eIF2Ba ${ }^{\mathrm{N} 208 \mathrm{Y}}$ was modestly reduced compared to WT $(14.5 \pm 0.9 \mathrm{~min}$; Fig. $5 \mathrm{~b})$ and this activity was responsive to ISRIB but not to F6P (11.2 \pm 0.3 min for ISRIB, $p=0.0026$ vs control; $13.3 \pm 0.8$ min for F6P, $p=0.25$ vs control). As we previously reported, eIF2B reconstituted with 
a

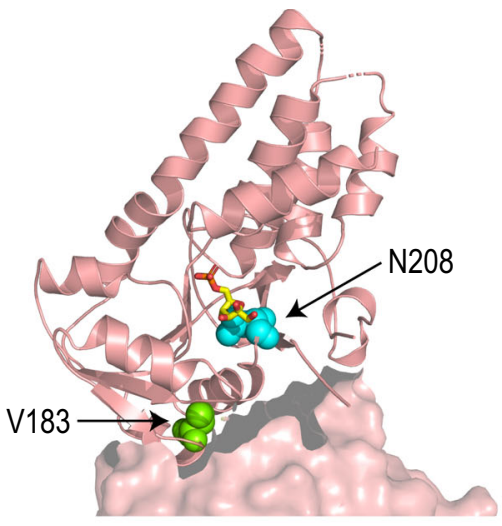

b

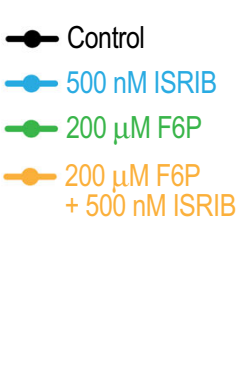

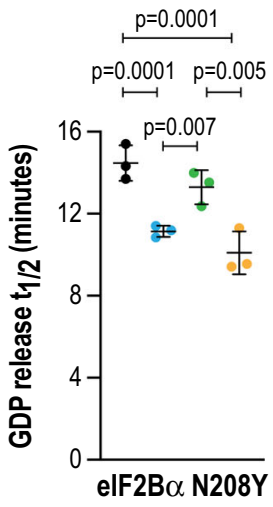

C
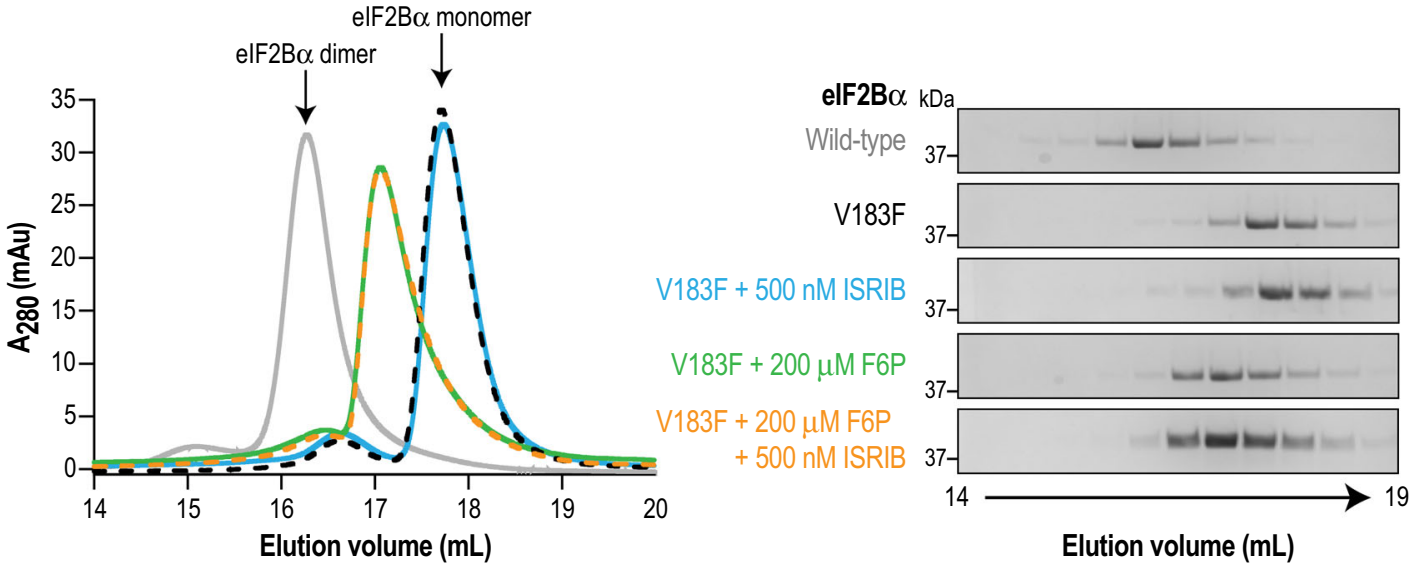

d
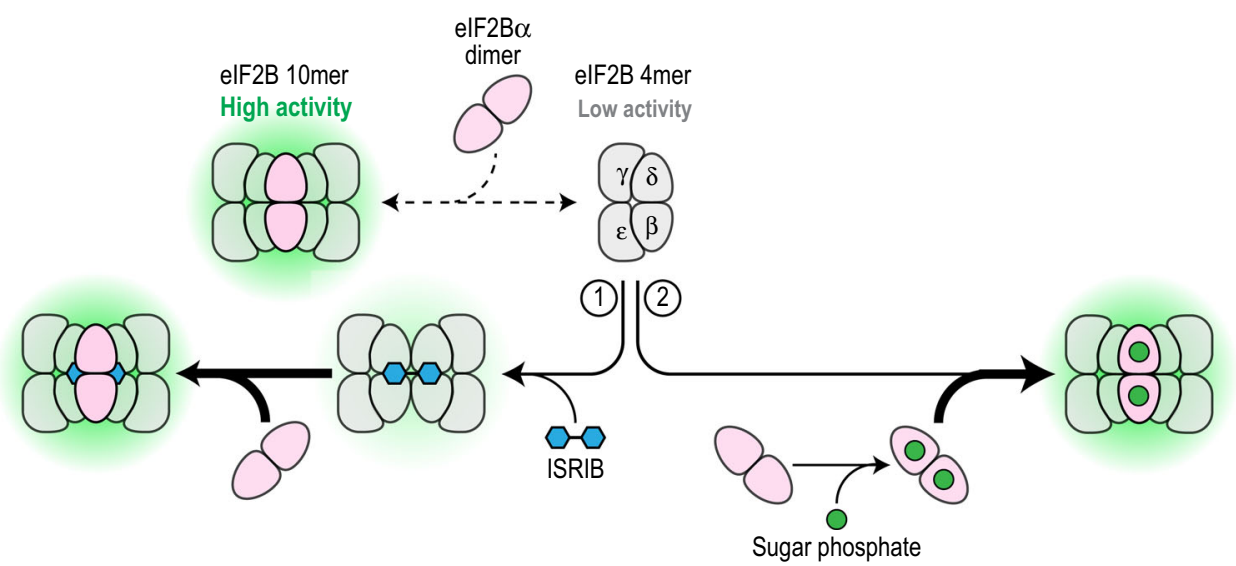

Fig. 5 F6P enhances decamer formation and activity of the VWM mutant elF2B $\boldsymbol{\alpha}^{\text {V183F }}$ but not elF2B $\boldsymbol{\alpha}^{\mathbf{N 2 0 8 Y}}$. a Close-up view of a single elF2B $\alpha$ monomer showing the positions of residues V183 (green) and N208 (cyan). F6P is shown in stick representation. N208 is within the binding pocket and V183 is positioned at the interface with another elF2B $\alpha$ subunit. $\mathbf{b}$ GDP release $t_{1 / 2}$ in a GEF assay using recombinant elF2B reconstituted with either elF2B $\alpha^{\mathrm{N} 208 Y}$ or elF2B ${ }^{\mathrm{V} 183 \mathrm{~F}}$. N208Y activity is stimulated by ISRIB (blue) but not F6P (green), whereas the reverse is true for V183F. Both mutants are stimulated by the combination of ISRIB and F6P (orange). Bars are mean \pm standard deviation of $n=3$ independent experiments of 3 technical replicates each. Statistical significance was tested by one-way ANOVA with Tukey's multiple testing correction. c Size-exclusion chromatography of purified recombinant wild-type or V183F elF2B $\alpha$ in the presence of ISRIB and/or F6P. UV absorbance chromatograms as well as Coomassie-stained fractions are shown. Data shown are representative of 3 independent replicates. Wild-type elF2B $\alpha$ is a dimer whereas elF2B $\alpha 183 \mathrm{~F}$ is a monomer, but is shifted towards a dimeric form by F6P. $\mathbf{d}$ Model depicting two distinct pathways to achieve elF2B decamerization and activation. Arrow thickness indicates the rate of a reaction occurring. The synthetic activator ISRIB bridges the elF2B $\beta / \delta$ interface to form an octamer, which then interacts with elF2B $\alpha_{2}$. Sugar phosphates bind to $\mathrm{elF} 2 \mathrm{~B} \alpha_{2}$ and promote its interaction with elF $2 \mathrm{~B}(\beta \delta \gamma \varepsilon)$ to form the holoenzyme.

eIF2Ba ${ }^{\mathrm{V} 183 \mathrm{~F}}$ exhibits markedly reduced basal GEF activity that is only partially restored by ISRIB $(21.2 \pm 0.5 \mathrm{~min}$ and $17.7 \pm 0.1$ min, respectively; Fig. 5b). Surprisingly, incubation with F6P caused a dramatic increase in the activity of V183F mutant eIF2B
(11.5 $\pm 0.1 \mathrm{~min})$. As with WT eIF2B, combining the two ligands did not yield further enhancement of activity.

A potential explanation for the striking rescue of eIF2Ba $\alpha^{\mathrm{V} 183 \mathrm{~F}}$ activity by F6P is through stabilization of the compromised $\alpha_{2}$ 
homodimer, which then promotes decamer formation. To evaluate this possibility, we subjected eIF2BaV183F to sizeexclusion chromatography and followed its fate after addition of F6P or ISRIB. Consistent with previous reports ${ }^{8}$, wild-type eIF2B $a$ migrates as a dimer whereas eIF2Ba $1183 \mathrm{~F}$ migrates as a monomer (Fig. 5c, gray line and dashed black line, respectively), highlighting the crippling effect of this mutation. Notably, F6P induced a partial shift from the monomer to the dimer peak, demonstrating a stabilizing effect on the VWM mutant. As expected, ISRIB had no effect on the a subunit.

To investigate the effects of F6P and ISRIB on V183F holoenzyme formation, we subjected eIF2 $\mathrm{Ba}$ V183F mutant lysates to sucrose gradient centrifugation. ISRIB and F6P promoted decamer formation at comparable levels (Supplementary Fig. 11c, d). We confirmed this observation by using a size-exclusion chromatography assay with recombinant complexes (Supplementary Fig. 11e). However, the higher sensitivity of the latter assay enabled us to detect a significant reduction of the eIF2BaV183F monomer peak in the presence of F6P. Collectively, the data indicate that dimerization of V183F eIF2B can be elicited by promoting eIF2B $\alpha^{\mathrm{V} 183 \mathrm{~F}}$ dimerization, or by stapling $\operatorname{eIF} 2 \mathrm{~B}(\beta \delta \gamma \varepsilon)$ subcomplexes.

As mentioned above, we were unable to generate viable cells harboring the eIF2 $\mathrm{Ba}^{\mathrm{N} 208 \mathrm{Y}}$ mutation. At face value, the $\mathrm{eIF} 2 \mathrm{Ba}^{\mathrm{N} 208 \mathrm{Y}}$ data suggest that recognition of sugar phosphates may be important in maintaining eIF2Ba stability and/or regulating eIF2B function in a physiological context. Inability to respond may be a possible route that leads to VWM disease, either by loss of specific activity or destabilization of the holoenzyme. However, even robust binding to metabolites is not sufficient if other aspects of protein stability are compromised, as evinced by the $\mathrm{IF} 2 \mathrm{Ba}{ }^{\mathrm{V} 183 \mathrm{~F}}$ mutant.

\section{Discussion}

eIF2B is unique among GEFs in its size and complexity, a property which has not been well understood. Although the GEF catalytic domain is located in the $\varepsilon$ subunit, formation of the decameric complex is necessary for full activity, as it forms a scaffold for interaction with its substrate eIF $2^{30-33}$. In previous work, we and others elucidated the mechanism by which synthetic eIF2B activators (ISRIB and 2BAct) directly staple the two halves of the eIF2B decamer together by bridging the regulatory $\beta$ and $\delta$ subunits ${ }^{9,10,12}$. More recently, ISRIB was shown to act as an allosteric antagonist of phospho-eIF $2 \alpha$ binding ${ }^{21,22}$. The evidence presented suggests that this is its primary mechanism of action in cells with high levels of eIF2B subunits, and importantly, sufficient levels of the decamer-promoting eIF2Ba dimer.

Here, beginning from unbiased efforts to identify eIF2B binders and activators, we showed that sugar phosphates occupy an ancestral ligand pocket in eIF2Ba, promoting holoenzyme decamerization. We present a working model to illustrate that activation of the eIF2B complex can now be achieved in two ways: Nature's solution, dating back billions of years, and one developed in the last decade through man-made chemistry (Fig. 5d). Perhaps owing to its site of action in eIF2Ba, we found that unlike ISRIB, sugar phosphates do not possess the ability to antagonize the interaction between eIF2B and phospho-eIF2 $\alpha$ in a biochemical experiment. Thus, these activators have overlapping but distinct mechanisms.

Although we have demonstrated a functional role for sugar phosphate binding in modulation of eIF2B decamerization and activity, the changes elicited by such an interaction remain unclear as we did not observe major structural shifts in the eIF2Ba structure upon ligand binding. Interpretation of the data are complicated by the fact that a true ligand-free "apo" structure of eIF2Ba does not exist-the solved structures contain a sulfate ion in the sugar phosphate binding pocket (an artifact of the crystallization buffer). Comparisons against existing decameric structures of eIF2B were also uninformative, as those structures already represent the stabilized state of the complex. Elucidation of these changes may require dynamic methods rather than the static "snapshots" obtained herein.

Pathogenic eIF2B partial loss-of-function mutations are present in all subunits. Three of these mutations that map to eIF2Ba provided us with important insights as to the role of sugar phosphates in eIF2B activation in vivo. N208Y and E198K are located in the sugar phosphate binding pocket and both abolish ligand binding. Both mutations had modestly reduced GEF activity and rendered eIF2B unresponsive to sugar phosphates. The knowledge that N208Y is a human disease-causing mutation suggests that occupation of this site may be important for eIF2B function. Similarly, in yeast, eIF2Ba ${ }^{\mathrm{E} 199 \mathrm{~K}}$ (equivalent to human E198K) was originally identified through screening as a constitutively depressing (i.e. ISR-activating) mutant ${ }^{34}$. An important caveat is that the N208Y mutation (N209Y in yeast) reportedly reduces the level of eIF2Ba in yeast ${ }^{35}$. Whereas eIF2Ba is dispensable for survival in yeast, it is essential in metazoans. This may be one explanation for our inability to generate human eIF2Ba ${ }^{\mathrm{N} 208 Y}$ knock-in mutant cells. Notably, we observed reduced eIF2B subunit levels in HEK293T cells with the eIF2$\mathrm{Ba}^{\mathrm{E} 198 \mathrm{~K}}$ knock-in mutation (Supplementary Fig. 8e). If the N208Y mutation does destabilize eIF2Ba in humans, we cannot deconvolve this effect from an additional effect on metabolite binding. It may also mean that sugar phosphate binding is obligate for the stability of the eIF2Ba subunit. However, clinical evidence implies that $\mathrm{eIF} 2 \mathrm{Ba}{ }^{\mathrm{N} 208 \mathrm{Y}}$ is viable as the only source of a subunit in a living human ${ }^{28}$, so compensatory mechanisms may exist or the genetic background of HEK293T cells may have been incompatible with this mutation.

In contrast to eIF2Ba $208 \mathrm{Y}$, the eIF2Ba ${ }^{\mathrm{V} 183 \mathrm{~F}}$ mutation disrupts formation of the $\alpha_{2}$ dimer and severely decreases basal GEF activity but is highly responsive to $\mathrm{F} 6 \mathrm{P}$ stimulation in vitro. Despite our finding that F6P can partially stabilize this mutant dimer, the pathogenicity of this mutation in vivo suggests that sugar phosphates may not always occupy the pocket in cells. Alternatively, it may mean that ligand binding is insufficient to rescue full eIF2B function in the full physiological context. A recent intriguing observation is the striking cell-type susceptibility of astrocytes to the hypomorphic alleles of eIF2 $\mathrm{B}^{13}$. The ISR is triggered early on in VWM disease progression only in these cells. It is tempting to speculate that the unique metabolic characteristic of astrocytes may result in lower occupancy by sugar phosphates and overall decreased GEF activity, which could exacerbate the effects of destabilized VWM mutant eIF2B complexes.

Our findings provide a facile explanation for the use of a metabolic enzyme as the eIF2B regulatory core. We propose that sugar phosphate binding may represent an ancient regulatory mechanism that directly couples carbon nutrient sensing to protein synthesis, predating the evolution of the panoply of stress-responsive eIF2 $\alpha$ kinases in higher eukaryotes. Intracellular free concentrations of sugar phosphates may vary depending on cell and tissue type, circadian rhythm, nutritional flux, metabolite microdomains and more - these may all impinge on eIF2B function in a physiological setting. It is likely that many cellular systems are governed by similar systems, such as the recently identified role of fructose-1,6-bisphosphate sensing by aldolase as a novel nucleotide-independent mechanism of AMPK regulation ${ }^{36}$. Another well-established allosteric regulator in the cell is fructose-2,6-bisphosphate, which undergoes significant changes in intracellular concentration in response to glucose uptake. We were unable to determine whether this sugar 
phosphate interacts with eIF2Ba due to lack of a commercial source, but it could be an ideal candidate for a physiological modulator of eIF2B activity.

In yeast, many metabolic enzymes are capable of undergoing dramatic and reversible conformational changes in response to nutrient status. Indeed, yeast eIF2B has been shown to form cellspanning fibrils, although the conditions for formation and ultimate function of these structures are debated ${ }^{37,38}$. Recent work suggests that eIF $2 \mathrm{~B} a$ is essential for the formation of these eIF2B bodies, and that VWM mutations (including N209Y) disrupt this process $^{39}$. We thus speculate that metabolite binding may be one possible mechanism to modulate these conformational shifts. It is conceivable that many similar mechanisms existed to support the most basic needs of early unicellular organisms-to grow when resources are abundant, and to quiesce during periods of scarcity. Our observation here that an eIF2Ba mutation abrogates its nutrient sensing ability and causes VWM disease in humans suggests the tantalizing possibility that this function may retain some importance even in complex multicellular organisms. It is possible that the pocket occupied by synthetic eIF2B activators may also serve as a binding site for as-yet-unidentified naturally occurring metabolites. This pocket may sense metabolites involved in a different pathway to couple protein synthesis capacity to other processes in the cell. Ultimately, understanding the role of ligand binding in control of eIF2B activity in vivo will be of utmost importance. Our in vitro data lay the groundwork for addressing these questions using more complex experimental systems.

\section{Methods}

Protein purification. The nucleotide sequence of eIF2Ba (Uniprot accession: Q14232) was synthesized by Genscript with a C-terminal TEV-avi-FLAG tag and cloned into a pET45b vector (Novagen) for expression. Point mutations of eIF2B $a$ were introduced by site-directed mutagenesis. All constructs were sequenced to verify the expected sequences.

Wild-type eIF2Ba and the mutants (V183F, E198K, N208Y) were expressed in BL21(DE3) cells (NEB). Cells were grown to a density of OD600 $=0.8$ and induced with $360 \mu \mathrm{M}$ IPTG. Cells were shaken in a beveled flask at $200 \mathrm{rpm}, 18^{\circ} \mathrm{C}$ for $16 \mathrm{~h}$. Cells were harvested by pelleting and resuspended in $25 \mathrm{mM}$ Tris, $150 \mathrm{mM} \mathrm{NaCl}$, 1 mM DTT, pH 8.0 + Halt protease inhibitor cocktail (Thermo Fisher). Cells were lysed by sonication and lysates were cleared by centrifugation at $33,000 \times g, 4{ }^{\circ} \mathrm{C}$, $1 \mathrm{~h}$. Supernatants were purified by FLAG affinity chromatography (Genscript). Target fractions were collected, and proteins were further purified on a Superdex200 column (GE Healthcare) using $20 \mathrm{mM}$ HEPES, $150 \mathrm{mM} \mathrm{NaCl}, 1 \mathrm{mM}$ DTT, pH 7.5. Purified proteins were concentrated and stored at $-80^{\circ} \mathrm{C}$ until further use. Purification of $\operatorname{eIF} 2 \mathrm{~B}\left(\beta \delta \gamma^{2}\right)$ and $\mathrm{eIF} 2$ was performed, as previously described $^{12}$.

\section{Compounds and antibodies. ISRIB was synthesized in-house as previously} described ${ }^{40}$. Metabolites were purchased from Millipore Sigma, Cayman Chemicals, Avanti Polar Lipids, Enamine or Combi-Blocks, Inc and prepared as $10 \mathrm{mM}$ stocks arrayed in 96 -deep well storage plates and stored at $-80^{\circ} \mathrm{C}$. Information on source and solvent for each metabolite is available in Supplementary Data 1 and 2. Metabolite accurate mass, adduct, purity, and optimal detection parameters were determined by independently assaying each metabolite by FIA-MS at $\mathrm{pH} 3,5,6.8$, and 9 in positive and negative mode ( 4 technical replicates) with interspersed blank injections on an Agilent 6550 QTOF MS platform. The optimal adduct, $\mathrm{pH}$, and polarity of each metabolite was considered to construct four unique metabolite screening pools validated by FIA-MS on Agilent 6550 QTOF MS and SCIEX X500R QTOF MS platforms.

The following antibodies were used in this study: eIF2B $\alpha$ (Proteintech \#18010-1-AP); eIF2B $\delta$ (Proteintech \#11332-1-AP); eIF2Be (Bethyl Labs \#A302556); eIF3a (Cell Signaling Technology \#3411); eIF2a (Cell Signaling \#5324); peIF2a (Cell Signaling \#3398); FLAG (Sigma \#F1804). All antibodies were used at 1:1000 dilution for detection.

MIDAS. Metabolites were combined into four defined screening pools. For each metabolite pool, $5 \mu \mathrm{L}$ of $\sim 960 \mu \mathrm{M}$ eIF2Ba was arrayed in triplicate across a 10 MWC 96-well microdialysis plate (SWISSCI) and sealed (protein chamber). To the reverse side, $300 \mu \mathrm{L}$ of $50 \mu \mathrm{M}$ per metabolite pool was aliquoted and sealed (metabolite chamber). Loaded dialysis plates were placed in the dark at $4{ }^{\circ} \mathrm{C}$ on a rotating shaker $(120 \mathrm{rpm})$ and incubated for $40 \mathrm{~h}$. Post-dialysis, metabolites were isolated from each chamber by cold methanol extraction. Each sample was analyzed in technical triplicate by FIA-MS on a SCIEX X500R QTOF MS with interspersed blanks injections. The input metabolite pools were assayed at the beginning, middle, and end of each MS method batch for QC and normalization.

MIDAS FIA-MS spectra were processed in SCIEX OS 1.6 software to determine metabolite abundances by integrating the mean area under the curve for each extracted ion chromatogram. For each dialysis replicate, $\log _{2}$ (fold change) for each metabolite was calculated as the difference between the $\log _{2}$ abundance in the protein chamber and metabolite chamber. For each technical triplicate, up to one outlier was removed using a $z$-score cutoff of five $(<0.1 \%$ of observations). The corrected technical replicates were collapsed to one mean fold-change summary per protein-metabolite pair. To remove fold-change variation that was not specific to a given metabolite-protein pair, the first three principal components of the total screening dataset were removed on a per metabolite pool basis by subtracting the projection of the first 3 principal components, creating $\log _{2}$ (corrected fold change) Protein-metabolite $\mathrm{z}$-scores were determined by comparing the target proteinmetabolite $\log _{2}$ (corrected fold change) to a no-signal model for that metabolite using measures of the central tendency (median) and standard deviation (extrapolated from the $25-75 \%$ quantiles), which are robust to the signals in the tails of a metabolite's fold-change distribution. Z-scores were false-discovery rate controlled using Storey's q-value (qvalue, R) and protein-metabolite interactions with $q$-values $<0.1$ were considered significant.

Differential scanning fluorimetry. The effect of metabolite binding on eIF2Ba $\mathrm{T}_{\mathrm{m}}$ was assessed using the Protein Thermal Shift assay (Thermo Fisher), following manufacturer's instructions. Briefly, reaction conditions for each $20 \mu \mathrm{L}$ reaction were: $1.1 \mu \mathrm{g}(1.5 \mu \mathrm{M})$ of eIF2Ba, $5 \mu$ Thermal Shift buffer, $1 \mathrm{X}$ Thermal Shift dye, and metabolites in dose response. Reactions were run in 384-well format on a QuantStudio 6 Flex instrument (Thermo Fisher). The resulting melt curves were analyzed using Protein Thermal Shift Software, and eIF2Ba $\mathrm{T}_{\mathrm{m}}$ s for each condition were calculated by fitting to the Boltzmann function.

Isothermal titration calorimetry. Interactions between recombinant protein and sugar phosphates were conducted with a MicroCal PEAQ-ITC (Malvern Panalytical Inc.). Experiments were performed in $25 \mathrm{mM}$ HEPES, $150 \mathrm{mM} \mathrm{NaCl}, \mathrm{pH} 7.5$ at $20^{\circ} \mathrm{C}$. The sugar phosphate concentration in the syringe was $1 \mathrm{mM}$ or $1.5 \mathrm{mM}$, and $\sim 3.5 \mu \mathrm{L}$ aliquots were injected into cells containing $300 \mu \mathrm{L}$ eIF2B $\alpha$ or eIF2B $(\beta \delta \gamma \varepsilon)$ at a concentration of $20-30 \mu \mathrm{M}$. The final titration curves were fitted using MicroCal ITC data analysis software, assuming a single binding site per monomer.

elF2B activity assays. Bodipy-FL-GDP-loaded eIF2 was used as a substrate for recombinant eIF2B. The assay was performed in 384-well plates. Reactions were read on a SpectraMax i3x plate reader (Molecular Devices) using the instrument's Softmax Pro software with the following parameters: plate temperature $=25^{\circ} \mathrm{C}$; excitation wavelength $=485 \mathrm{~nm}(15 \mathrm{~nm}$ width $)$; emission wavelength $=535 \mathrm{~nm}$ ( $25 \mathrm{~nm}$ width); read duration $=30 \mathrm{~min}$ at $45 \mathrm{~s}$ intervals. Data were analyzed in Prism. GDP release half-lives were calculated by fitting single-exponential decay curves.

For unbiased screening, MIDAS metabolite libraries at $10 \mathrm{mM}$ stock concentration were dispensed into wells of the assay plate using an Echo Acoustic Liquid Handler (Labcyte). In a final assay volume of $10 \mu \mathrm{L} /$ well, the following conditions were kept constant: $25 \mathrm{nM}$ Bodipy-FL-GDP-loaded eIF2, $4 \mathrm{nM}$ phospho-eIF2, $0.1 \mathrm{mM}$ GDP, $1 \mathrm{mg} / \mathrm{mL}$ BSA, $1 \mathrm{nM}$ eIF2B, $10 \mu \mathrm{M}$ metabolite. A single well was run for each metabolite. For targeted experiments with recombinant wild-type and mutant eIF2Ba, the following conditions were used: $50 \mathrm{nM}$ BodipyFL-GDP-loaded eIF2, $0.1 \mathrm{mM}$ GDP, $1 \mathrm{mg} / \mathrm{ml}$ BSA, $20 \mathrm{nM}$ eIF2B. For targeted experiments with HEK293T lysates, the following conditions were used: $50 \mathrm{nM}$ Bodipy-FL-GDP-loaded eIF2, $0.1 \mathrm{mM} \mathrm{GDP}, 1 \mathrm{mg} / \mathrm{ml} \mathrm{BSA}, 0.1$ or $0.3 \mathrm{mg} / \mathrm{ml}$ lysates.

\section{Cryo-EM data collection and image processing. eIF2Ba-F6P and eIF2B $(\beta \delta \gamma \varepsilon)$} were mixed in a $\sim 1.5: 1$ molar ratio and incubated on ice for $1 \mathrm{~h}$. Prior to grid preparation, the protein mixture was diluted with $25 \mathrm{mM} \mathrm{HEPES,} 100 \mathrm{mM} \mathrm{KCl}$, $2 \mathrm{mM} \mathrm{MgCl}_{2}, 1 \mathrm{mM}$ DTT, pH 7.5 to a final concentration of $\sim 0.3 \mathrm{mg} / \mathrm{mL}$.

A $3 \mu \mathrm{L}$ drop of the sample was applied to a 1.2/1.3 UltrAuFoil grid (Quantifoil) that had been plasma-cleaned for $10-40 \mathrm{~s}$ using a $25 \% \mathrm{O}_{2} / 75 \% \mathrm{Ar}$ mixture in a Solarus 950 Plasma Cleaner (Gatan). Grids were plunge-frozen in liquid ethane using the following settings on a Vitrobot Mark IV (Thermo Fisher Scientific): blot time $4-6 \mathrm{~s}, 4^{\circ} \mathrm{C}, 100 \%$ humidity. The grids were imaged using an FEI Titan Krios (Hillsboro, Oregon) transmission electron microscope operated at $300 \mathrm{kV}$ and equipped with a Volta phase plate and Gatan K2 Summit direct detector placed at the end of a GIF Quantum 967 LS imaging filter, operating with a slit width of $20 \mathrm{eV}$ (Gatan, Inc.). Automated data collection was performed with Leginon software at a nominal magnification of $\times 130,000$, corresponding to a pixel size of $1.04 \AA^{41}$. A total of 4506 movies were recorded using a nominal defocus range of -1.0 to $-2.4 \mu \mathrm{m}$. Exposures were fractionated into 30 frames with an exposure rate of $7.6 \mathrm{e}^{-} / \mathrm{pixel} / \mathrm{s}$ and total exposure of $44.3 \mathrm{e}^{-} / \AA^{2}$. An additional dataset of 1567 movies was acquired at $30^{\circ}$ tilt $^{42}$. Data collection and image processing parameters for tilted and untilted data were identical.

Movie frames were motion-corrected and dose-weighted using MotionCor $2^{43}$ Further image processing was carried out in cryoSPARC ${ }^{44}$. CTF parameters were estimated from the dose-weighted aligned movie frames with Patch CTF. The 
images acquired at $0^{\circ}$ and $30^{\circ}$ tilt were pooled together. Projections of the cryo-EM structure of $H$. sapiens eIF2B decamer (EMD-7443) ${ }^{10}$ were generated using EMAN and were subsequently used as templates for particle picking. From 6073 total images collected, 1865 were rejected prior to $2 \mathrm{D} / 3 \mathrm{D}$ classification in cryoSPARC. The 736,044 particles from the remaining 4208 images were subjected to both $2 \mathrm{D}$ classification and $3 \mathrm{D}$ heterogenous refinement simultaneously. Multiple rounds of heterogeneous and homogeneous refinement were performed using the eIF2B map low-pass filtered to $25 \AA$ as an initial reference. During each round of $3 \mathrm{D}$ classification, only one of the models appeared to have the correct size corresponding to the intact eIF2B complex, whereas the other classes were too small to be the complex of interest. This 3D class had clear high-resolution features and was directly refined from that point, obviating the need for $2 \mathrm{D}$ classification for particle sorting. This was followed by several additional rounds of heterogeneous refinement and non-uniform refinement with $\mathrm{C} 2$ symmetry imposed. Subsequently, the map was refined by two rounds of global and per-particle CTF optimization, resulting in a final map with a resolution of $2.9 \AA$ using the goldstandard FSC $=0.143$ criteria.

Rigid-body docking of the coordinates of the H. sapiens eIF2B (PDB: 6CAJ) $)^{10}$ into the reconstructed EM density map showed reasonably good fit. Several rounds of manual model adjustment in COOT and refinement in phenix.real_space_refine were applied. The initial maps revealed noticeable variability in map resolution, showing the highest resolution at the center of the complex (for eIF2B $\alpha / \beta / \delta$ ) and much lower resolution at the periphery of the complex. The resolution was lowest for the eIF2B $\gamma / \varepsilon$ subunits, suggesting a higher amount of disorder/flexibility in this region. During the model building and refinement phase, a small density feature corresponding to F6P was observed only in the eIF2Ba subunit in the area that M6P has been previously modeled in the X-ray crystal structure. Analysis and model validation were performed with the aid of MOLPROBITY and COOT and Phenix validation tool. Data collection and processing statistics are provided in Supplementary Table 1 .

Crystallization and structure determination. The avi-FLAG tag on eIF2Ba was removed by TEV protease (NEB) prior to crystallization. For co-crystallization, eIF2B $\alpha$ was incubated with $8 \mathrm{mM}$ mannose-6-phosphate on ice for $1 \mathrm{~h}$. Crystals of eIF2B $\alpha$ in complex with mannose-6-phosphate were grown by sitting-drop vapor diffusion method at $18{ }^{\circ} \mathrm{C}$ for 5 days. The protein complex $(\sim 5 \mathrm{mg} / \mathrm{ml})$ was crystallized by mixing $1.5 \mu \mathrm{L}$ of the protein solution with $1.5 \mu \mathrm{L}$ of reservoir solution (12\% PEG 4000, $100 \mathrm{mM}$ sodium acetate, $100 \mathrm{mM}$ ammonium sulfate, $0.5 \%$ octylbeta-glucoside, $\mathrm{pH} 4.6$ ), and equilibrated over $360 \mu \mathrm{L}$ of the reservoir solution.

Crystals belonged to monoclinic space group P21 with the following unit cell parameters: $a=71.2 \AA, b=155.5 \AA, c=140.11 \AA$, and $\alpha=\gamma=90^{\circ}, \beta=103.9^{\circ}$. Prior to data collection, crystals were cryo-protected by addition of $25 \%$ glycerol (final concentration) to the crystallization drop. X-ray diffraction data were collected on the IMCA beamline at beamline 17-ID in the facilities of the Industrial Macromolecular Crystallography Association Collaborative Access Team (IMCACAT) at the Advanced Photon Source, Argonne National Laboratory. Data were reduced and scaled using autoPROC software. A previously reported eIF2Ba structure (PDB: 3ECS) was used as a starting model for iterative rounds of map fitting and refinement using the programs COOT, Buster, Refmac and Phenix. Data collection and refinement statistics are provided in Supplementary Table 2.

Generation of mutant cell lines. The eIF2Ba $a^{\mathrm{E} 198 \mathrm{~K}}$ mutation was introduced into HEK293T cells using the Alt-R CRISPR-Cas9 System (IDT). cRNA sequences were chosen with the IDT design tool to allow for CRISPR cutting near the E198 site. To introduce the point mutation, a 150-bp DNA donor template for homologydirected repair (HDR) was synthesized to include the G > A point mutation of interest, as well as silent mutations to prevent further Cas9 cutting.

To form CRISPR gRNA complex, equimolar amounts of crRNA and tracrRNA labeled with Atto550 were annealed by heating to $95^{\circ} \mathrm{C}$ for $5 \mathrm{~min}$ followed by cooling to room temperature. RNP complex was formed by incubating gRNA complex with Alt-R S.p. HiFi Cas9 Nuclease V3 (IDT). RNP complex, $100 \mu \mathrm{M}$ HDR template, and $100 \mu \mathrm{M}$ Alt-R Cas9 Electroporation Enhancer (IDT) were delivered to cells with a Lonza 4D-Nucleofector, using the 96-well SF reagent kit and program SF-130 following vendor instructions. Nucleofected cells were incubated in media containing $30 \mu \mathrm{M}$ Alt-R HDR Enhancer (IDT). After $48 \mathrm{~h}$, Atto550-positive cells were single-sorted into 96-well plates using a BD Biosciences FACSAria Fusion. Clones were expanded and genomic DNA was sequenced to confirm introduction of the mutant allele. A similar protocol was employed to generate HEK293T cells with eIF2B $\beta$ tagged with a C-terminal FLAG peptide at the endogenous locus. The eIF2Ba ${ }^{\mathrm{V} 183 \mathrm{~F}}$ HEK293T cell line was previously described ${ }^{12}$.

Sucrose gradients. WT or eIF2Ba mutant HEK293T cells were harvested and lysed in $50 \mathrm{mM}$ HEPES, $400 \mathrm{mM} \mathrm{KCl}, 1 \mathrm{mM}$ DTT, $4 \mathrm{mM} \mathrm{Mg}(\mathrm{OAc})_{2}$, $\mathrm{pH} 7.5+0.5 \%$ Triton-X-100 + EDTA-free protease inhibitor cocktail (Roche) on ice for $30 \mathrm{~min}$. Lysates were cleared by centrifugation at $48,000 \times g, 4^{\circ} \mathrm{C}$ for $30 \mathrm{~min}$. $100 \mu \mathrm{L}$ of each cleared lysate was loaded onto a 5-20\% sucrose gradient prepared in a $13 \times 51 \mathrm{~mm}$ ultracentrifuge tube using a Gradient Station (Biocomp) and centrifuged in a SW55 Ti rotor at $152,000 \times g, 4^{\circ} \mathrm{C}$ for $12 \mathrm{~h}$. After centrifugation, $24 \times 200 \mu \mathrm{L}$ fractions were manually collected from the top to bottom of each gradient. Each fraction was subjected to methanol/chloroform precipitation and SDS-PAGE followed by Western Blot with the indicated antibodies. Blots were imaged on a ChemiDoc MP (BioRad), and band intensities were quantified using the instrument's Image Lab software. Each value was then divided by total intensity obtained across all fractions to determine percent enrichment of each subunit in each fraction.

Size-exclusion chromatography. Purified proteins were thawed and centrifuged at $20,000 \times g, 10 \mathrm{~min}, 4^{\circ} \mathrm{C}$ to remove any potential precipitate. Concentrations of eIF $2 \mathrm{~B}(\beta \delta \gamma \varepsilon)$ and eIF $2 \mathrm{~B} \alpha$ were normalized to $2 \mu \mathrm{M}$ and $2.4 \mu \mathrm{M}$, respectively. $0.2 \mathrm{~mL}$ of each mixture was injected onto a Superose 6 Increase 10/300 column connected to an AKTA Pure 25 FPLC system (GE Healthcare). The system was run at $0.5 \mathrm{~mL} /$ min for $1 \mathrm{~h}$ using $25 \mathrm{mM}$ HEPES, $200 \mathrm{mM} \mathrm{KCl}, 2 \mathrm{mM} \mathrm{MgCl}_{2}, 1 \mathrm{mM}$ DTT, pH 7.5 as the mobile phase. For conditions with ISRIB, the protein samples and mobile phase were supplemented with $500 \mathrm{nM}$ ISRIB. For conditions with F6P, the protein samples and mobile phase were supplemented with $200 \mu \mathrm{M}$ F6P. UV 280 measurements were obtained directly by the instrument.

Phospho-elF2 $\alpha$ co-immunoprecipitation. HEK293T cells endogenously expressing eIF2B $\beta$ with a C-terminal FLAG tag were maintained in DMEM High Glucose (Corning CellGro) supplemented with 10\% FBS (Gibco) and antibiotic-antimycotic (Gibco). Upon reaching 90\% confluency, cells were treated with either DMSO or $100 \mathrm{nM}$ thapsigargin (Sigma) for $4 \mathrm{~h}$. Cells were harvested and lysed in $25 \mathrm{mM}$ HEPES, $150 \mathrm{mM} \mathrm{KCl}, 1 \% \mathrm{NP}-40,1 \mathrm{mM}$ EDTA, pH 7.4 + Complete protease inhibitor cocktail (Roche) and PhosStop (Roche) on ice for $20 \mathrm{~min}$. Lysates were cleared by centrifugation at $18,000 \times g, 20 \mathrm{~min}, 4^{\circ} \mathrm{C}$, and transferred to Eppendorf tubes containing anti-FLAG M2 magnetic beads (Sigma). Mixtures were incubated overnight at $4{ }^{\circ} \mathrm{C}$. To test the effect of ligands, these were added to the mixtures and incubated for an additional $2 \mathrm{~h}$ at $4^{\circ} \mathrm{C}$. Beads were washed three times with lysis buffer, eluted with $1 \times$ LDS buffer (Invitrogen), and subjected to SDS-PAGE followed by Western Blot with the indicated antibodies.

Reporting summary. Further information on research design is available in the Nature Research Reporting Summary linked to this article.

\section{Data availability}

The data generated or analyzed in the current study are available within the article, supplementary information, or from the corresponding authors upon reasonable request. The structures reported in this work have been deposited in public repositories with the following accession codes: eIF2B-F6P cryo-EM structure: PDB 7KMF, EMDB EMD22924; eIF2Ba-M6P X-ray crystal structure: PDB 7KMA. Source data are provided with this paper.

Received: 28 October 2020; Accepted: 19 May 2021; Published online: 08 June 2021

\section{References}

1. Hinnebusch, A. G. \& Lorsch, J. R. The mechanism of eukaryotic translation initiation: new insights and challenges. Cold Spring Harb Perspect Biol. 4, a011544 (2012)

2. Wek, R. C., Jiang, H.-Y. \& Anthony, T. G. Coping with stress: eIF2 kinases and translational control. Biochemical Soc. Trans. 34, 7-11 (2006).

3. Donnelly, N., Gorman, A. M., Gupta, S. \& Samali, A. The eIF2a kinases: their structures and functions. Cell Mol. Life Sci. 70, 3493-3511 (2013).

4. Harding, H. P. et al. Regulated translation initiation controls stress-induced gene expression in mammalian cells. Mol. Cell 6, 1099-1108 (2000).

5. Krishnamoorthy, T., Pavitt, G. D., Zhang, F., Dever, T. E. \& Hinnebusch, A. G. Tight binding of the phosphorylated subunit of initiation factor 2 (eIF2) to the regulatory subunits of guanine nucleotide exchange factor eIF2B is required for inhibition of translation initiation. Mol. Cell. Biol. 21, 5018-5030 (2001).

6. Yang, W. \& Hinnebusch, A. G. Identification of a regulatory subcomplex in the guanine nucleotide exchange factor eIF2B that mediates inhibition by phosphorylated eIF2. Mol. Cell. Biol. 16, 6603-6616 (1996).

7. Kashiwagi, K. et al. Crystal structure of eukaryotic translation initiation factor 2B. Nature 531, 122-125 (2016).

8. Wortham, N. C., Martinez, M., Gordiyenko, Y., Robinson, C. V. \& Proud, C G. Analysis of the subunit organization of the eIF2B complex reveals new insights into its structure and regulation. FASEB J. 28, 2225-2237 (2014).

9. Zyryanova, A. F. et al. Binding of ISRIB reveals a regulatory site in the nucleotide exchange factor eIF2B. Science 359, 1533-1536 (2018).

10. Tsai, J. C. et al. Structure of the nucleotide exchange factor eIF2B reveals mechanism of memory-enhancing molecule. Science 359, eaaq0939 (2018)

11. Sidrauski, C. et al. Pharmacological dimerization and activation of the exchange factor eIF2B antagonizes the integrated stress response. eLife 4, e07314 (2015) 
12. Wong, Y. L. et al. The small molecule ISRIB rescues the stability and activity of vanishing white matter disease eIF2B mutant complexes. eLife 7, 51 (2018).

13. Wong, Y. L. et al. eIF2B activator prevents neurological defects caused by a chronic integrated stress response. eLife 8, 1867 (2019).

14. Nakamura, A. et al. Dynamic, ligand-dependent conformational change triggers reaction of ribose-1,5-bisphosphate isomerase from Thermococcus kodakarensis KOD1. J. Biol. Chem. 287, 20784-20796 (2012).

15. Bogorad, A. M. et al. Insights into the architecture of the eIF2B $\alpha / \beta / \delta$ regulatory subcomplex. Biochemistry 53, 3432-3445 (2014).

16. Kuhle, B., Eulig, N. K. \& Ficner, R. Architecture of the eIF2B regulatory subcomplex and its implications for the regulation of guanine nucleotide exchange on eIF2. Nucleic Acids Res. 43, 9994-10014 (2015).

17. Krishna, K. H. \& Kumar, M. S. Molecular evolution and functional divergence of eukaryotic translation initiation factor 2-alpha kinases. PLOS ONE 13, e0194335 (2018).

18. Roussou, I., Thireos, G. \& Hauge, B. M. Transcriptional-translational regulatory circuit in Saccharomyces cerevisiae which involves the GCN4 transcriptional activator and the GCN2 protein kinase. Mol. Cell. Biol. 8, 2132-2139 (1988).

19. Gross, M., Rubino, M. S. \& Starn, T. K. Regulation of protein synthesis in rabbit reticulocyte lysate. Glucose 6-phosphate is required to maintain the activity of eukaryotic initiation factor (eIF)-2B by a mechanism that is independent of the phosphorylation of eIF-2 alpha. J. Biol. Chem. 263, 12486-12492 (1988).

20. Dholakia, J. N., Mueser, T. C., Woodley, C. L., Parkhurst, L. J. \& Wahba, A. J. The association of NADPH with the guanine nucleotide exchange factor from rabbit reticulocytes: a role of pyridine dinucleotides in eukaryotic polypeptide chain initiation. Proc. Natl Acad. Sci. 83, 6746-6750 (1986).

21. Schoof, M. et al. eIF2B conformation and assembly state regulates the integrated stress response. eLife 10, e65703 (2021).

22. Zyryanova, A. F. et al. ISRIB blunts the integrated stress response by allosterically antagonising the inhibitory effect of phosphorylated eIF2 on eIF2B. Mol. Cell 81, 88-103.e6 (2021).

23. Orsak, T. et al. Revealing the allosterome: systematic identification of metabolite-protein interactions. Biochemistry 51, 225-232 (2012).

24. Wishart, D. S. et al. HMDB 4.0: the human metabolome database for 2018. Nucleic Acids Res. 46, D608-D617 (2018).

25. Sekine, Y. et al. Stress responses. Mutations in a translation initiation factor identify the target of a memory-enhancing compound. Science 348, 1027-1030 (2015).

26. Tamura, H. et al. Crystal structure of 5-methylthioribose 1-phosphate isomerase product complex from Bacillus subtilis: implications for catalytic mechanism. Protein Sci. 17, 126-135 (2008).

27. Hiyama, T. B., Ito, T., Imataka, H. \& Yokoyama, S. Crystal structure of the alpha subunit of human translation initiation factor 2B. J. Mol. Biol. 392 937-951 (2009).

28. van der Knaap, M. S. et al. Mutations in each of the five subunits of translation initiation factor eIF2B can cause leukoencephalopathy with vanishing white matter. Ann. Neurol. 51, 264-270 (2002).

29. Ohlenbusch, A. et al. Identification of ten novel mutations in patients with eIF2B-related disorders. Hum. Mutat. 25, 411 (2005).

30. Kenner, L. R. et al. eIF2B-catalyzed nucleotide exchange and phosphoregulation by the integrated stress response. Science 364, 491-495 (2019).

31. Kashiwagi, K. et al. Structural basis for eIF2B inhibition in integrated stress response. Science 364, 495-499 (2019).

32. Adomavicius, T. et al. The structural basis of translational control by eIF2 phosphorylation. Nat. Commun. 10, 2136 (2019).

33. Gordiyenko, Y., Llácer, J. L. \& Ramakrishnan, V. Structural basis for the inhibition of translation through eIF2 $\alpha$ phosphorylation. Nat. Commun. 10 2640 (2019).

34. Hannig, E. M., Williams, N. P., Wek, R. C. \& Hinnebusch, A. G. The translational activator GCN3 functions downstream from GCN1 and GCN2 in the regulatory pathway that couples GCN4 expression to amino acid availability in Saccharomyces cerevisiae. Genetics 126, 549-562 (1990).

35. Richardson, J. P., Mohammad, S. S. \& Pavitt, G. D. Mutations causing childhood ataxia with central nervous system hypomyelination reduce eukaryotic initiation factor 2B complex formation and activity. Mol. Cell. Biol. 24, 2352-2363 (2004).

36. Zhang, C.-S. et al. Fructose-1,6-bisphosphate and aldolase mediate glucose sensing by AMPK. Nature 548, 112-116 (2017).

37. Marinia, G., Nüskea, E., Lenga, W., Albertia, S. \& Pigino, G. Reorganization of budding yeast cytoplasm upon energy depletion. Mol. Biol. Cell mbcE20020125 https://doi.org/10.1091/mbc.E20-02-0125 (2020).

38. Nüske, E. et al. Filament formation by the translation factor eIF2B regulates protein synthesis in starved cells. Biol. Open bio.046391 https://doi.org/ 10.1242/bio.046391 (2020)
39. Norris, K. et al. Mutational analysis of the alpha subunit of eIF2B provides insights into the role of eIF2B bodies in translational control and VWM disease. J. Biol. Chem. 296, 100207 (2020).

40. Sidrauski, C. et al. Pharmacological brake-release of mRNA translation enhances cognitive memory. eLife 2, e00498 (2013).

41. Suloway, C. et al. Automated molecular microscopy: the new Leginon system. J. Struct. Biol. 151, 41-60 (2005).

42. Tan, Y. Z. et al. Addressing preferred specimen orientation in single-particle cryo-EM through tilting. Nat. Meth 14, 793-796 (2017).

43. Zheng, S. Q. et al. MotionCor2: anisotropic correction of beam-induced motion for improved cryo-electron microscopy. Nat. Meth 14, 331-332 (2017).

44. Punjani, A., Rubinstein, J. L., Fleet, D. J. \& Brubaker, M. A. cryoSPARC: algorithms for rapid unsupervised cryo-EM structure determination. Nat. Meth 14, 290-296 (2017).

\section{Acknowledgements}

We thank our AbbVie colleagues Corey Smith, Kent Baker, Soo Ro, and Chaohong Sun for help with the purification of recombinant eIF2 and eIF2B. We thank our Calico colleagues John Wang, Janani Sridar, Crystal Ghosh and Roy Ghosh for experimental support. We thank Mandy Janssen from NanoImaging Services for assistance with cryoEM data collection and processing. For X-ray crystallography, the use of the IMCA-CAT beamline 17-ID at the Advanced Photon Source was supported by the companies of the Industrial Macromolecular Crystallography Association through a contract with Hauptman-Woodward Medical Research Institute. Use of the Advanced Photon Source was supported by the US Department of Energy, Office of Science, Office of Basic Energy Sciences, under contract \#DE-AC02-06CH1135. This work was supported by Calico Life Sciences LLC.

\section{Author contributions}

These authors contributed equally: Q.H. and J.-M.H. These authors jointly supervised this work: Y.L.W. and C.S. Y.L.W. and C.S. conceptualized and supervised the work. V.S.S., D.E., and J.R. provided additional supervision. Q.H., J.H., B.P.N., K.G.H., C.R., S.H., L.L., R.J., and Y.L.W. performed experiments. Y.L.W. and C.S. wrote the manuscript. All authors contributed to review and editing.

\section{Competing interests}

Q.H., J.H., C.R., S.H., L.L., D.E., and Y.L.W. are employees of Calico Life Sciences LLC and declare no other competing interests. C.S. is an employee of Calico Life Sciences LLC and is an inventor on U.S. Patent 9708247 describing ISRIB and its analogs. Rights to the invention have been licensed to Calico Life Sciences LLC from the University of California, San Francisco. B.P.N., V.S.S., and R.J. are employees of AbbVie and declare no other competing interests. The remaining authors declare no competing interests.

\section{Additional information}

Supplementary information The online version contains supplementary material available at https://doi.org/10.1038/s41467-021-23836-z.

Correspondence and requests for materials should be addressed to Y.L.W. or C.S.

Peer review information Nature Communications thanks Assen Marintchev, Alan Roseman, and other, anonymous, reviewers for their contributions to the peer review of this work.

Reprints and permission information is available at http://www.nature.com/reprints

Publisher's note Springer Nature remains neutral with regard to jurisdictional claims in published maps and institutional affiliations.

Open Access This article is licensed under a Creative Commons Attribution 4.0 International License, which permits use, sharing, adaptation, distribution and reproduction in any medium or format, as long as you give appropriate credit to the original author(s) and the source, provide a link to the Creative Commons license, and indicate if changes were made. The images or other third party material in this article are included in the article's Creative Commons license, unless indicated otherwise in a credit line to the material. If material is not included in the article's Creative Commons license and your intended use is not permitted by statutory regulation or exceeds the permitted use, you will need to obtain permission directly from the copyright holder. To view a copy of this license, visit http://creativecommons.org/ licenses/by/4.0/.

(C) The Author(s) 2021 